
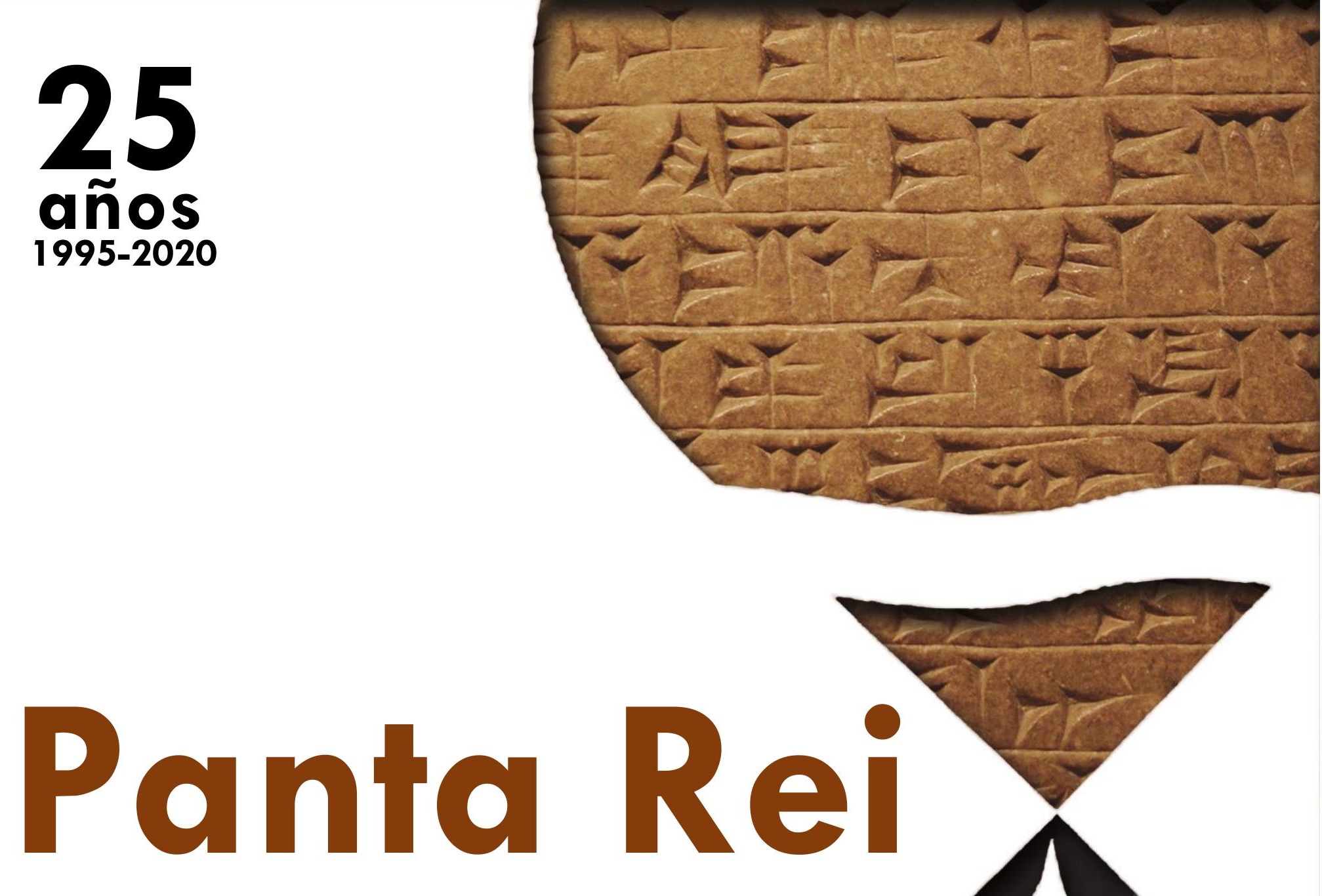

Revista digital de Historia y didáctica de la Historia 


\section{0 (2)}

Revista anual

Fecha de inicio: 1995

Revista Panta Rei. pantarei@um.es

\section{Edita:}

Centro de Estudios del Próximo Oriente y la Antigüedad Tardía - CEPOAT

Edificio Universitario Saavedra Fajardo.

Universidad de Murcia

C/ Actor Isidoro Máiquez, 9

30007 - MURCIA - ESPAÑA

Teléfono: $(+34) 868883890$

cepoat@um.es

Web: www.um.es/cepoat/pantarei

Ediciones de la Universidad de Murcia - EDITUM

Edificio Pleiades. Campus de Espinardo.

Universidad de Murcia

C/ Campus, s/n

30100 - MURCIA - ESPAÑA

Teléfono: $(+34) 868883013$

editum@um.es

Web: https://www.um.es/web/editum/

En portada: Montaje elaborado a partir de fotografía de una inscripción sumeria del British Museum (Wikimedia) (arriba) y fotografía de Miguel Martínez Sánchez (abajo)

Edición 2020 (2)

ISSNe: 2386-8864

ISSN: $1136-2464$
Responsables de los textos: sus autores.

Responsable de la presente edición: Consejo Editorial de Panta Rei.

Depósito legal: MU-966-1995 


\section{CONSEJO DE REDACCIÓN}

Coordinador editorial

Egea Vivancos, Alejandro [Didáctica de las Ciencias Sociales, Universidad de Murcia]

Secretaria

Arias Ferrer, Laura [Didáctica de las Ciencias

Sociales, Universidad de Murcia]

Editores

Jiménez Vialás, Helena [Historia Antigua, Universidad de Murcia]

Martínez Gil, Tània [Didáctica de las Ciencias

Sociales, Universidad de Barcelona]

Meseguer Gil, Antonio José [Historiador, Profesor de

Secundaria]

Ortiz García, Jónatan [Arqueología, Universidad de

Alcalá de Henares]

Romero Molero, Alberto [Arqueología, Universidad

Isabel I]

Sáez Giménez, David Omar [Historiador, Profesor

de Secundaria]

Sáez Rosenkranz, Isidora V. [Didáctica de las

Ciencias Sociales, Universidad de Barcelona]

Sánchez Mondejar, Celso Miguel [Arqueólogo,

Patrimonio Inteligente]

Responsable informático

Martínez García, José Javier [CEPOAT, Universidad de Murcia]

Responsables de traducción y corrección lingüística Martínez Martínez, Cristina [Profesora de Secundaria, Sociedad Española de Lenguas Modernas] Albaladejo Albaladejo, Sara [ISEN-Universidad de Murcia]

\section{CONSEJO ASESOR}

Adroher Auroux, Andrés María [Arqueología, Universidad de Granada]

Albero Muñoz, $M^{a}$ del Mar [H. ${ }^{a}$ del Arte, Universidad de Murcia]

Alia Miranda, Francisco [Historia Contemporánea, UCLM]

Arciniega García, Luis [Historia del Arte, Universidad de Valencia]

Barrio Barrio, Juan Antonio [Historia Medieval,

Universidad de Alicante]

Castellano i Solé, Núria [Egiptología, Schola

Didàctica Activa S.L.]

Chapman, Arthur [History Education, University

College of London, Reino Unido]

Cid López, Rosa María [Historia Antigua, Universidad de Oviedo]

Cobacho López, Ángel [Derecho, Universidad de Murcia]

Cuenca López, José María [Didáctica de las Ciencias Sociales, Universidad de Huelva]
Egea Bruno, Pedro M. ${ }^{a}$ [Historia Contemporánea, Universidad de Murcia]

Feijoo Martínez, Santiago [Arqueología, Consorcio

Ciudad Monumental de Mérida]

García Atienzar, Gabriel [Prehistoria, Universidad de

Alicante]

Ginestí Rosell, Anna [Filología Clásica, Katholische

Universität Eichstätt-Ingolstadt]

González Monfort, Neus [Didáctica de las

Ciencias Sociales, Universidad Autónoma de

Barcelona]

González Soutelo, Silvia [Arqueología, Universidad de Vigo]

Haber Uriarte, María [Prehistoria, Universidad de Murcia]

Hernández de la Fuente, David [Filología Clásica, Universidad Complutense]

Hutson, Scott R. [Anthropology, University of Kentucky, EEUU]

Igual Luis, David [Historia Medieval, UCLM]

Irigoyen López, Antonio [Historia Moderna,

Universidad de Murcia]

Jover Maestre, Francisco Javier [Prehistoria,

Universidad de Alicante]

Mahony, Simon [Digital Humanities, University College of London, Reino Unido]

Marsilla de Pascual, Francisco Reyes [Técnicas

historiográficas, Universidad de Murcia]

Martínez-Burgos García, Palma [H. ${ }^{a}$ del Arte, UCLM]

Mathis, Christian [Didaktik der Geschichte, PH Zürich]

Miralles Maldonado, José Carlos [Filología Clásica,

Universidad de Murcia]

Molina Gómez, José Antonio [Historia Antigua,

Universidad de Murcia]

Mónica Ghirardi [Historia Moderna, Universidad

Nacional de Córdoba, Argentina]

Navarro Espinach, Germán [Historia Medieval,

Universidad de Zaragoza]

Noguera Celdrán, José Miguel [Arqueología,

Universidad de Murcia]

Ortiz Heras, Manuel [Historia Contemporánea, UCLM]

Panzram, Sabine [Historia Antigua, Universität

Hamburg]

Pérez Molina, Miguel Emilio [Filología Clásica,

Universidad de Murcia]

Prados Martínez, Fernando [Arqueología,

Universidad de Alicante]

Sánchez lbáñez, Raquel [Didáctica de las Ciencias

Sociales, Universidad de Murcia]

Sancho Gómez, Miguel Pablo [Educación, UCAM]

Victoria Moreno, Diego [Historia Contemporánea,

UNED]

Vilar García, María José [Historia Contemporánea,

Universidad de Murcia]

Vivas Sainz, Inmaculada [H. ${ }^{a}$ del Arte, UNED]

Zamora López, José Ángel [Próximo Oriente Antiguo,

CCHS-CSIC] 



\section{Índice}

1995-2020. 25 años de Panta Rei

Equipo editorial

La Historia social aplicada a la antigua Mesopotamia: cambios historiográficos y nuevas vías de 9 investigación

Josué Javier Justel Vicente

A Look Back into Ancient Egyptian Linguistic Studies (c. 1995-2019)

Carlos Gracia Zamacona

La infancia en la antigua Mesopotamia: 25 años de investigación

Daniel Justel Vicente

Conflictos armados y su tratamiento en educación. Análisis de la producción científica de los últimos

25 años en la Web of Science

Naiara Vicent, Janire Castrillo, Alex Ibañez-Etxeberria y Leire Albas

La investigación en pensamiento histórico. Un estudio a través de las tesis doctorales de Ciencias

Sociales (1995-2020)

Álvaro Chaparro Sainz, M.a del Mar Felices de la Fuente y Laura Triviño Cabrera

Las narrativas nacionales en la enseñanza y el aprendizaje de la historia: Una revisión sobre los 149 libros de texto y las narrativas de los estudiantes

César López Rodríguez

La historia de las personas: reflexiones desde la historiografía y de la didáctica de las ciencias 167 sociales durante los últimos 25 años

Mariona Massip Sabater, Jordi Castellví Mata y Joan Pagès Blanch †

Panorámica de la trilogía cine, historia y educación en España (1995-2020)

Concha Fuentes-Moreno y Alba Ambrós-Pallarés 



\title{
Conflictos armados y su tratamiento en educación. Análisis de la producción científica de los últimos 25 años en la Web of Science
}

\author{
Armed Conflicts and their Treatment in Education: Analysis of the \\ Scientific Production of the Last 25 Years in the Web of Science \\ Naiara Vicent \\ Universidad del País Vasco (UPV/EHU) \\ naiara.vicent@ehu.eus \\ 0000-0002-2678-0098 \\ Alex lbañez-Etxeberria \\ Universidad del País Vasco (UPV/EHU) \\ alex.ibanez@ehu.eus \\ 0000-0001-9495-5155 \\ Janire Castrillo \\ Universidad del País Vasco (UPV/EHU) \\ janire.castrillo@ehu.eus \\ 0000-0002-8128-9090 \\ Leire Albas \\ Universidad del País Vasco (UPV/EHU) \\ leirealbas@gmail.com \\ 0000-0002-7240-9978
}

Recibido: $28 / 02 / 2020$

Aceptado: $27 / 06 / 2020$

\section{Resumen}

En este trabajo se realiza una revisión bibliométrica sobre estudios que abordan el tratamiento de conflictos armados de corte político en educación, con ánimo de obtener un esbozo de lo que se está realizando actualmente en el panorama internacional. Para ello, se ha realizado una búsqueda y análisis de estudios asociados a la didáctica de las ciencias sociales y la educación para la ciudadanía en la Web of Science (WOS). La muestra final, compuesta de 128 estudios, denota un crecimiento de investigaciones sobre la temática en los últimos años y el uso de múltiples metodologías y enfoques. Expone también un rico panorama en cuanto a escenarios de conflicto y temáticas tratadas. Apunta, en último término, a la utilidad de llevar estas temáticas a las aulas de cara a mejorar la convivencia y a promocionar la paz y la reconciliación en las sociedades afectadas por conflictos violentos.

\section{Palabras clave}

Ciencias Sociales, Educación para la ciudadanía, Temas controvertidos, Paz,

\section{Abstract}

In this work, a bibliometric review is carried out on studies that deal with the treatment of politically-motivated armed conflicts in education, with the aim of obtaining an outline of what is currently being done on the international scene. To this end, a search and analysis of studies associated with the teaching of social sciences and citizenship education in Web of Science (WOS) has been undertaken. The final sample, comprising 128 studies, denotes a growth in research on the subject in recent years and the use of multiple methodologies and approaches. It also presents a rich panorama in terms of conflict scenarios and issues addressed. Finally, it points out the usefulness of bringing these issues into the classroom in order to promote the peaceful coexistence and reconciliation in societies affected by violent conflicts.

\section{Keywords}

Social Sciences, Citizenship Education, Controversial Issues, Peace, Historiography. Historiografía.

Para citar este artículo: Vicent, N., Castrillo, J., Ibañez-Etxeberria, A. y Albas, L. (2020). Conflictos armados y su tratamiento en educación. Análisis de la producción científica de los últimos 25 años en la Web of Science. Panta Rei: revista digital de Historia y didáctica de la Historia, 55-91. doi: $10.6018 /$ pantarei.445721 


\section{Introducción}

La formación de una ciudadanía participativa y democrática viene siendo impulsada desde diferentes países, así como desde la Unión Europea, a través de los currículos escolares (Eurydice, 2012). Hace tiempo que desde la didáctica de las ciencias sociales se viene reivindicando la utilidad de éstas en la formación de una ciudadanía crítica y comprometida en la mejora de la sociedad (de Alba Fernández, García Pérez y Santisteban Fernández, 2012). Para ello, resulta necesario trabajar el análisis crítico de las fuentes de información y la argumentación desde la comprensión de nuestras sociedades actuales. Igualmente, bajo el marco democrático actual, abordar la convivencia y la educación en valores resulta imprescindible, debiendo situarse el respeto hacia los derechos humanos como eje vertebrador de la educación para la ciudadanía (Banks, 2017).

Los debates sociales de actualidad presentan un gran potencial para trabajar las ciencias sociales en el aula desde la perspectiva indicada en el párrafo anterior. En el currículo del Reino Unido, la enseñanza de la ciudadanía recoge la controversia como contenido, con el fin de recopilar, analizar e interpretar datos y entender las limitaciones, validez y fiabilidad de los mismos (Qualifications and Curriculum Authority, 2006, citado por Pineda-Alonso, 2015). Sin embargo, esta realidad no es generalizable, y como afirman Rubin y Cervinkova (2019), los intentos globales de trabajar la convivencia democrática desde la educación han dejado de lado el tratamiento de aquellos temas difíciles del pasado, fuentes de divergencia y recuerdos históricos conflictivos de las comunidades. Estas mismas autoras reconocen la dificultad de enfrentarse a historias difíciles, pero destacan la oportunidad que ofrece para comprender mejor el presente y construir una versión más justa e inclusiva de la democracia.

Jiménez Martínez y Felices de la Fuente (2018) proponen trabajar cuestiones controvertidas en tres fases: aproximación a la problemática; construcción de narrativas críticas y fundamentadas; y producción final. En la primera fase, el alumnado recopila la información necesaria para poder crear su propia opinión desde el conocimiento, la cual será representada a través de la segunda fase. En ésta, surgirán opiniones diversas, dando la posibilidad de trabajar la convivencia desde el respeto, pudiendo defender cualquier postura e ideología, pero siempre respetando los derechos humanos (López Facal y Santidrián, 2011).

El debate que conllevan estos temas supone una dificultad para parte del profesorado ya que, como señala Santisteban Fernández (2019), su papel no se ha de corresponder al de un espectador neutral, sino al de acompañante del alumnado en la indagación y discusión en libertad. Así, si bien la propuesta de incluir en la programación problemas sociales existe desde la primera mitad del siglo XX, todavía hoy en día hay grandes reticencias a hacerlo. Un estudio realizado en Chile (Toledo Jofré, Magendzo Kolstrein, Gutiérrez Gianella, e Iglesias Segura, 2015), demostraba el rechazo del profesorado de secundaria a abordar temas controvertidos, evitando el conflicto y mostrando temor a manifestar su opinión, siendo los temas identificados como más complicados, aquellos relacionados con la violación de los derechos fundamentales de las personas. 


\section{La oportunidad de trabajar temas controvertidos en el País Vasco}

Uno de estos temas que ha emergido con fuerza en el País Vasco es el de las secuelas producidas en la sociedad tras más de 50 años de violencia y terrorismo. Tras el fin de ETA, el País Vasco se ha visto inmerso en una nueva etapa de su historia en la que, como sociedad, tiene que hacer frente a la recuperación de la convivencia entre sus ciudadanos/as. La sociedad vasca ha vivido durante las últimas décadas una situación anómala, marcada por la violencia, el sufrimiento, el miedo, las emociones y por fuertes tensiones que han dado lugar a numerosos conflictos entre la ciudadanía. La gravedad del asunto ha dado lugar a un proceso de polarización entre sectores de la sociedad que, aun hoy en día, sigue existiendo. Sin embargo, la juventud se siente en cierta manera lejana a este problema. Si bien muestra un alto interés por conocer lo ocurrido, cuenta con un significativo nivel de desconocimiento y reconoce su insatisfacción hacia la información recibida (Usón González, 2017).

En el País Vasco, las instituciones y asociaciones, vienen abordando un impulso a las políticas en torno a esta cuestión. En concreto, en el ámbito de las políticas públicas impulsadas por la Secretaría de paz, convivencia y derechos humanos del gobierno regional, se han ido desarrollando una serie de planes (Gobierno Vasco, 2008; 2010; 2013; 2017), entre los que encontramos propuestas didácticas como el Módulo Educativo Adi-Adian. Aprendizajes de dignidad humana, convivencia y empatía mediante una experiencia de escucha de testimonios de víctimas (Gobierno Vasco, s.f.). Este programa, cuyo eje central es la incorporación del testimonio de víctimas de la violencia terrorista y contraterrorista al aula, ofrece la oportunidad de trabajar un tema controvertido, desde los contenidos específicos de las materias relacionadas con las ciencias sociales y los valores sociales y cívicos recogidos en el currículo vasco de Educación Básica ${ }^{1}$.

Este programa se ha implementado, al principio de modo experimental, y actualmente integrado como contenido del área de didáctica de las ciencias sociales, en la formación inicial del profesorado de Educación Infantil, Primaria y Secundaria en la Universidad del País Vasco UPVEHU (Albas, lbañez-Etxeberria, Echeberria y Vicent, en prensa; Aranguren e lbañez-Etxeberria, 2020). Se ha entendido, además del derecho a conocer de la juventud vasca, que nuestro alumnado, como futuros educadores, tendrá responsabilidades educativas en que la situación vivida se interprete desde una visión crítica, y que no se vuelva a repetir. Asimismo, el tema ofrecía la oportunidad de conocer de manera práctica cómo se puede trabajar un tema controvertido en el aula.

Pero no vivimos solos, y en este mundo interconectado, somos conscientes de que la problemática del tratamiento en las aulas de la violencia de motivación política ejercida y/o soportada sobre parte o la totalidad de la población, es un hecho global, complejo y poliédrico, que afecta a más conjuntos de ciudadanos/as, y que se extiende por todo el mundo. Por ello, dentro del marco del Proyecto de Innovación Educativa Memoria y convivencia, en este trabajo planteamos la necesidad de conocer el tratamiento que se ha dado a dicho fenómeno, y conocer otras experiencias e implementaciones educativas que hayan arrojado luz a este tema. Para lo cual, planteamos el análisis de la literatura científica que, desde la enseñanza-aprendizaje de las ciencias sociales y/o la educación para la ciudadanía, haya tratado y comunicado las acciones educativas

1 Decreto 236/2015, de 22 de diciembre, por el que se establece el currículo de la Educación Básica y se implanta en la Comunidad Autónoma del País Vasco (BOPV, 15-01-2016). https://www.euskadi.eus/decretos-curricularesde-la-capv/web01-a3hbhezi/es/ 
realizadas en torno a problemas controvertidos de motivación política, y más en concreto, a aquellos relacionados con conflictos de origen político que han derivado en violencia armada continuada. Para ello, y siguiendo la línea marcada por otros estudios recientes desarrollados desde el ámbito de la didáctica de las ciencias sociales (Calaf, Gutiérrez Berciano y Suárez Suárez, 2020; Fontal e lbañez-Etxeberria, 2017; Gómez-Carrasco, López-Facal y RodríguezMedina, 2019), se va procede a la realización de un estudio bibliométrico, cuyo objetivo específico es identificar en la literatura del área, a través del análisis de la producción bibliográfica recogida durante los últimos 25 años en la colección principal de Web of Science (WOS), las temáticas y respuestas que se han dado desde la educación al tratamiento de esa huella de violencia en las sociedades que la han padecido.

\section{Método}

Este es un estudio exploratorio de tipo descriptivo en base a categorías. Se ha realizado sobre análisis de textos (títulos, palabras clave y resúmenes) y sobre los datos estadísticos de frecuencias y porcentajes proporcionados por las herramientas internas de tipo cuantitativo que ofrece WOS.

Como universo en el que realizar nuestra investigación y obtener una muestra representativa de publicaciones de trabajos científicos, se ha optado por limitar la herramienta de búsqueda a la colección principal (Core Collection) de WOS. Las razones que nos han llevado a la elección de esta base de datos son: los indicios de calidad que presentan los artículos que recoge, la amplitud de la misma, su carácter internacional, la duplicidad inter-bases y las ya citadas herramientas que ofrece para conocer algunos datos estadísticos en torno a la selección de publicaciones realizada.

Así, entre los meses de noviembre de 2019 y enero 2020, se ha rastreado la base de datos en busca de las publicaciones que, durante los últimos 25 años (entre 1995 y 2019), hayan tratado el tema que nos interesa. Para dar con ellas, se han definido una serie de términos de búsqueda, los cuales se han aplicado al título, resumen y palabras clave (propuestas por el/la autor/a y keywords plus que ofrece la propia herramienta). Dado que las revistas recogidas en WOS cuentan, independientemente del idioma en que se haya publicado, con el título, el resumen y las palabras clave en inglés, éste ha sido el único idioma utilizado para la localización de trabajos.

Siguiendo el método OEPE (Fontal, 2016), una vez realizada la búsqueda, se ha llevado a cabo un proceso de inclusión y exclusión buscando inventariar únicamente aquellos casos susceptibles de ser analizados.

\subsection{Definición de los términos de búsqueda}

Para la elección de los términos de búsqueda se ha partido de los tesauros ERIC y UNESCO. A través de los mismos, se ha ido conformando un listado, partiendo de unos primeros términos de referencia a partir de los cuales se ha ido derivando a otros secundarios. Estos términos se han diferenciados en dos bloques. El primero de ellos hace referencia a los ámbitos curriculares desde los que se desarrolla el análisis: didáctica de las ciencias sociales y educación para la ciudadanía. Los segundos están relacionados con la temática concreta que se pretende analizar desde los 
campos anteriores, habiéndose buscado tanto conceptos que puedan tener vinculación con el conflicto armado como con la superación del mismo.

Tras la búsqueda en los tesauros, se ha procedido a la elaboración del listado definitivo a emplear (Tabla 1). En la toma de decisiones, se han priorizado los conceptos propuestos por la UNESCO para aquellas cuestiones más relacionadas con los derechos humanos. Por el contrario, para aquellos de carácter educativo se han considerado los propuestos por ERIC. Por otro lado, se ha procedido a la depuración de algunos sinónimos propuestos por los tesauros que no se correspondían de manera clara con la temática a tratar. También, se han eliminado los términos específicos, seleccionando únicamente el término genérico (por ejemplo: guerra), salvo en el caso de los conflictos, entre los que únicamente se ha elegido el término conflicto armado.

\section{Tabla 1}

Listado de términos de búsqueda a emplear en la localización de publicaciones

\begin{tabular}{|c|c|c|c|}
\hline & & UNESCO & ERIC \\
\hline \multirow[t]{4}{*}{ Ámbito curricular } & $\begin{array}{l}\text { Didáctica de las } \\
\text { ciencias sociales }\end{array}$ & $\begin{array}{l}\text { Social studies } \\
\text { Social science(s) } \\
\text { education }\end{array}$ & Social Studies \\
\hline & & History education & History Instruction \\
\hline & & $\begin{array}{l}\text { Humanities education } \\
\text { Humanities }\end{array}$ & Humanities Instruction \\
\hline & $\begin{array}{l}\text { Educación para la } \\
\text { ciudadanía }\end{array}$ & $\begin{array}{l}\text { Citizenship education } \\
\text { Civic education }\end{array}$ & Citizenship Education \\
\hline \multirow[t]{7}{*}{ Ámbito temático } & Desde el conflicto & $\begin{array}{l}\text { Terrorism } \\
\text { Torture }\end{array}$ & Terrorism \\
\hline & & Violence & Violence \\
\hline & & War & War \\
\hline & & Armed conflict & \\
\hline & & & Victims \\
\hline & Desde la pacificación & Peace education & \\
\hline & & $\begin{array}{l}\text { Disarmament education } \\
\text { Human rights education }\end{array}$ & \\
\hline
\end{tabular}

Fuente: elaboración propia.

Cabe especificar, que en el caso de los términos humanities y social studies, se busca identificar aquellas acepciones vinculadas al sistema educativo. 


\subsection{Obłención de la muestra}

Una vez definido el listado de los términos de búsqueda, se ha procedido a la localización de publicaciones en WOS, para lo cual se han cruzado los términos referidos al ámbito curricular con los referidos a la temática a tratar (Tabla 1 ).

Para la selección de los casos que conforman la muestra definitiva, se ha aplicado al universo de casos obtenido de la búsqueda ( $\mathrm{N}=339$ ) una serie de criterios de inclusión y exclusión (Tabla 3), los cuales han sido aplicados en el orden que se desglosa a continuación (Tabla 2), obteniendo una muestra final de 128 publicaciones (Anexo l).

\section{Tabla 2}

Fases de cribado para la obtención de la muestra final de publicaciones

\begin{tabular}{|c|c|c|c|c|}
\hline Fase de cribado & & Fase 1 & Fase 2 & Fase 3 \\
\hline Criterios empleados & & E1 y E2 & I1/E3 & 12 e $13 /$ E4 y E5 \\
\hline Muestra & $N=339$ & $n=261$ & $n=243$ & $n=128$ \\
\hline
\end{tabular}

Fuente: elaboración propia.

En la primera fase, se han eliminado los resultados que presentaban algún tipo de error técnico en relación a las palabras de búsqueda (E1). Se trata de errores derivados de la presencia de elementos que distorsionan el significado de los conceptos de búsqueda: comas que las deslindan, por ejemplo: "History, Education" en lugar de History Education; palabras añadidas que cambian el significado del término, por ejemplo: "social studies of medicine"; o casos en los que el concepto forma parte de un nombre propio sin aportar nada a la temática a tratar, como "Join University Council for Social Studies". Asimismo, en este primer paso de cribado se han excluido también todos aquellos casos que no cuenta con resumen (E2) y que, por lo tanto, no ofrecen información suficiente para interpretar su contenido y poder pasar el resto de filtros de inclusión y exclusión.

\section{Tabla 3}

Criterios de inclusión y exclusión para la obtención de la muestra final

Fase Criterio de inclusión Criterio de exclusión

1

$2 \quad 11$-Condición indispensable: Tener alguna vinculación con la didáctica de las ciencias sociales - la educación para la ciudadanía.
E1: Las palabras clave de búsqueda no son lo que se esperaba, cuentan con otro significado o forman parte de un nombre propio.

E2: No cuenta con abstract, por lo que no ofrece información suficiente para poder pasar los filtros de inclusión y exclusión.

E3: No se cumple el criterio indispensable de inclusión 11. Es decir, no tiene vinculación alguna con la didáctica de las ciencias sociales o la educación para la ciudadanía. 
12: Se cita un conflicto armado de motivación política alargado en el tiempo.

13: No se cita ningún conflicto, pero se puede interpretar un contexto en el que ha existido un clima de violencia armada continuada de motivación política.
E4: Disertaciones en las que se cita un conflicto armado de motivación política alargado en el tiempo, pero de manera genérica o anecdótica.

E5: No se identifica un contexto en el que ha existido un clima de violencia armada continuada de motivación política.

Fuente: elaboración propia.

Una vez completada esa primera criba, se ha procedido a la lectura y análisis detenido de los casos restantes. En esta segunda fase, se han descartado aquellos casos que no cumplen la condición indispensable (I1) de estar relacionados con la didáctica de las ciencias sociales o la educación para la ciudadanía (E3). Entre ellos, se han encontrado propuestas en las que se citaban los social studies pero no desde un punto de vista educativo; o publicaciones en las que los términos se citan de manera anecdótica.

En la tercera y última fase, se han excluido los casos que, si bien citan conflictos armados, únicamente se refieren a ellos de manera genérica, por ejemplo, disertando sobre el concepto guerra; o anecdótica, muchas veces como hito temporal a partir del cual se da un cambio (E4). Esto ha ocurrido en varias ocasiones, por ejemplo, al citar las guerras mundiales como revulsivo para el cambio en la educación cívica o enseñanza de la historia. También se han eliminado las publicaciones que, a través de su título, resumen y palabras clave, no se enmarcan en un contexto en el que haya existido un clima de violencia armada continuada de motivación política (E5). Este es el caso, por ejemplo, de una serie de artículos referidos al bullying. Por último, se ha empleado el criterio de inclusión 13, manteniendo en la muestra los casos en los que, a pesar de que no se explicita ningún contexto de violencia armada continuada de motivación política, se pude interpretar la existencia del mismo.

\subsection{Herramientas de recogida y análisis de datos}

Esta investigación combina el análisis estadístico con el análisis de textos. A través de un análisis de frecuencias de las publicaciones que conforman la muestra, se quiere conocer el perfil general de los trabajos que se vienen realizando durante los últimos 25 años en torno al tratamiento educativo de los conflictos armados de motivación política que han perdurado en el tiempo. La combinación de la información obtenida por cada uno de los análisis nos permitirá acercarnos al conocimiento de la situación actual en la que se encuentra la literatura científica, así como identificar las publicaciones, autores principales $y / o$ fuentes de referencia al respecto.

Para el análisis estadístico, se han empleado las herramientas informe de citas e análisis de resultados que ofrece WOS. A partir del informe de citas se puede conocer el índice h y el $n .^{\circ}$ de citas recibidas por la muestra y por cada una de las referencias; $n .{ }^{\circ}$ de citas por año de la totalidad de la muestra; 0 n..$^{\circ}$ de publicaciones que citan los trabajos de la muestra. A través del análisis de resultados se han obtenido frecuencias para las siguientes variables: autores y país al que se adscriben; títulos de las revistas (sources), colecciones de libros y congresos (meeting) en 
los que se han presentado; año e idioma de publicación; y áreas de investigación en la que se enmarcan.

Para el análisis de texto, se ha realizado una lectura pormenorizada de una serie de datos que WOS ofrece para cada publicación. La de carácter más general es aquella que recoge si cuenta con acceso abierto o no. Mientras que aquellas a través de las que se quieren conocer cuestiones más relacionadas con la temática que queremos abordar son: título, abstract, palabras clave del autor y keywords plus. La información recibida a través de éstas se ha recogido en una serie de variables predefinidas, en las que, en los casos que corresponde, se han creado una serie de categorías de respuesta (Tabla 4).

La información referida a los criterios de inclusión y exclusión, así como aquella referida al análisis cualitativo, se ha recogido a través del programa Excel 2016, con el cual se han obtenido las frecuencias para cada variable que cuenta con categorías.

En el caso del análisis estadístico realizado a través de las herramientas que ofrece WOS, hay casos en los que existe más de una posibilidad de respuesta, por lo que, en ocasiones, los resultados dan un número mayor a la muestra. Por contra, al categorizar los datos derivados del análisis textual, se ha creado la opción de respuesta multicategoría en los casos en los que se recoge más de una categoría. Si bien se han tenido en cuenta como una categoría aparte, se ha revisado la información recogida, aportando datos en los casos más relevantes. Esto se ha realizado en la mayoría de variables, buscando información más pormenorizada a través de la lectura del abstract.

\section{Tabla 4}

Categorización de las variables de creación propia

\begin{tabular}{|c|c|c|}
\hline Categorías & & \\
\hline Ámbito curricular & Didáctica de las ciencias sociales & \\
\hline desde el que se & Educación para la ciudadanía & \\
\hline & Ambas & \\
\hline $\begin{array}{l}\text { Lugar donde se } \\
\text { desarrolla la } \\
\text { experiencia }\end{array}$ & Respuesta abierta & \\
\hline $\begin{array}{l}\text { ¿Se ha realizado } \\
\text { alguna investigación? }\end{array}$ & Tipo de investigación & $\begin{array}{l}\text { Cuantitativa } \\
\text { Cualitativa }\end{array}$ \\
\hline $\begin{array}{l}\text { Respuesta: } \\
\text { No/Sí }\end{array}$ & & Mixta \\
\hline & $\begin{array}{l}\text { Estrategia/Técnicas de } \\
\text { investigación }\end{array}$ & Respuesta abierta \\
\hline $\begin{array}{l}\text { ¿Se presenta alguna } \\
\text { propuesta educativa? }\end{array}$ & Tipo de propuesta educativa & $\begin{array}{l}\text { Política educativa } \\
\text { Programa }\end{array}$ \\
\hline Respuesta: & & Material \\
\hline & & Proyecto/experiencia \\
\hline & & Multicategoría \\
\hline & Etapa educativa & Educación Infantil \\
\hline
\end{tabular}




\begin{tabular}{|c|c|c|c|}
\hline \multirow{4}{*}{$\begin{array}{l}\text { ¿Se enmarca en la } \\
\text { educación formal? } \\
\text { Respuesta: } \\
\text { No/Sí }\end{array}$} & & \multicolumn{2}{|l|}{ Educación Primaria } \\
\hline & & \multicolumn{2}{|l|}{ Educación Secundaria } \\
\hline & & \multicolumn{2}{|l|}{ Educación Superior } \\
\hline & & \multicolumn{2}{|l|}{ Multicategoría } \\
\hline ¿Se explicita algún & Nombre del conflicto & \multicolumn{2}{|l|}{ Respuesta abierta } \\
\hline concreto? & Territorio en el que ocurre & \multicolumn{2}{|l|}{ Respuesta abierta } \\
\hline Respuesta: & & & \\
\hline \multirow[t]{11}{*}{ No/Sí } & Año en el que finaliza & \multicolumn{2}{|l|}{ Respuesta abierta } \\
\hline & Periodo en el que finaliza & Hace más de 100 años & $\begin{array}{l}\text { No cuenta con } \\
\text { supervivientes }\end{array}$ \\
\hline & & Hace 50-100 años & \\
\hline & & Hace $25-50$ años & Cuenta con \\
\hline & & Últimos 25 años & supervivientes \\
\hline & & Sigue vigente & \\
\hline & & Multicategoría & \\
\hline & Tipo de conflicto armado & \multicolumn{2}{|l|}{$\begin{array}{l}\text { Conflicto universal } \\
\text { Conflicto entre países }\end{array}$} \\
\hline & & \multicolumn{2}{|l|}{ Guerra Civil } \\
\hline & & \multicolumn{2}{|l|}{ Terrorismo yihadista } \\
\hline & & \multicolumn{2}{|l|}{$\begin{array}{l}\text { Grupos armados } \\
\text { Represión contra } \\
\text { minorías } \\
\text { Conflicto colonial, post- } \\
\text { colonial } \\
\text { Multicategoría }\end{array}$} \\
\hline
\end{tabular}

Fuente: elaboración propia.

\section{Resultados}

Si comenzamos por la autoría de las publicaciones, Waghid (2009 y con Davids, 2015 y 2016) y Yemini (con Dvir, Bonshtein y Natur, 2018; Goren 2016; Goren y Maxwell, 2019), son los investigadores que cuentan con un mayor número de publicaciones $(n=3)$, trabajando cuestiones referidas a Sudáfrica e Israel respectivamente. Otros/as 10 autores/as, a saber, Akar (2016; y con Albretch 2017), Baliqi (2017; 2018), Bellino (2015; 2016), Davids, Elwick (con Jerome, 2019; Lee, 2019), Emerson (2018a y b), Goren, Guerra-Sua (2019; y con Bickmore y Kaderi, 2017), Philippou (2009; y con Theodorou, 2019) y Skaras (2019; y con Breidlid, 2016) cuentan con 2 publicaciones cada uno/a, repitiendo temática (guerras civiles del Líbano, Kosovo, Guatemala y Sudan del Sur; conflictos sociales en Pakistán; conflicto colombiano y árabe-israelí; prevención del terrorismo en Reino Unido; e identidad chipriota). El resto presentan una única publicación en la muestra. Sin embargo, cabe decir que algunos/as de éstos/as, como Nygren (con Jonhsrud, 2018), Bickmore, Finkel (con Horowitz y Rojo-Mendoza, 2012), Rubin (2016) ○ Sen (2019), contaban con otros artículos sobre educación para la ciudadanía eliminados en la tercera fase de cribado, por no hacer referencia directa a propuestas vinculadas a un conflicto armado de motivación política concreto. 
En cuanto a los medios de difusión, las publicaciones no presentan unos canales principales claros, no habiendo una preponderancia, ya que ninguna revista supera el $4 \%$ de la producción. En esta dispersión, destacan las recogidas en la Tabla 5.

\section{Tabla 5}

Principales revistas en las que se ha publicado

\begin{tabular}{lcc}
\hline Nombre de la revista & $\mathrm{N}$ & $\%$ \\
\hline Education Citizenship and Social Justice & 5 & 3,9 \\
Compare a Journal of Comparative and International Education & 4 & 3,1 \\
Journal of Peace Education & 4 & 3,1 \\
British Journal of Educational Studies & 3 & 2,3 \\
Comparative Education & 3 & 2,3 \\
Education as Change & 3 & 2,3 \\
Journal of Curriculum Studies & 3 & 2,3 \\
\hline
\end{tabular}

Fuente: elaboración propia a partir de los datos de WOS.

Solo se citan 5 aportaciones a congresos relevantes y cada uno cuenta con una aportación, por lo que no se ha detectado ningún foro referente. Lo mismo ocurre con las colecciones de libros, ya que encontramos 2 trabajos recogidos en la misma colección, pero son capítulos de un mismo libro.

En relación a las áreas de investigación, la mayoría de trabajos, con mucha diferencia, se recogen bajo la categoría de educación e investigación educativa, aunque también en otras áreas de ciencias sociales (Tabla 6), pudiendo, en ocasiones, combinarse más de una categoría.

\section{Tabla 6}

Principales áreas de investigación en las que se engloban las publicaciones

\begin{tabular}{lcc}
\hline Áreas de investigación & $\mathrm{N}$ & $\%$ \\
\hline Education Educational Research & 79 & 61,7 \\
Government Law & 15 & 11,7 \\
History & 14 & 10,9 \\
International Relations & 6 & 4,7 \\
Area Studies & 5 & 3,9 \\
Ethnic Studies & 4 & 3,1 \\
Social Sciences Other Topics & 4 & 3,1 \\
Religion & 3 & 2,3 \\
Sociology & 3 & 2,3
\end{tabular}


Arts Humanities Other Topics

Cultural Studies

Fuente: elaboración propia a partir de los datos de WOS.

En cuanto al idioma de publicación, la mayoría de los trabajos ( $\mathrm{n}=105 ; 82 \%$ ) han sido publicados en inglés, seguido muy de lejos por aquellas publicaciones en español $(n=17 ; 13,9 \%)$. También se encuentran 2 en francés $(1,6 \%)$ y otras 2 en turco $(1,6 \%)$; otra en afrikáans $(0,8 \%)$ y una última en portugués $(0,8 \%)$.

Estos datos se relacionan con la procedencia de los/las autores/as. Como se puede ver en el Figura 1, el mayor número de publicaciones se ha realizado por personal adscrito a instituciones de Estados Unidos; seguido de Inglaterra; Colombia y España; y en menor medida, Israel; Sudáfrica; Canadá; Australia, Alemania y Suecia; Chipre, Líbano, China, Corea del Sur y Turquía. Otros países que presentan autoría son Chile, Indonesia, Irlanda, Kosovo, Irlanda, Noruega y Nueva Zelanda, que cuentan con 2 publicaciones; y otra serie de lugares mínimamente representados como Argentina, Bélgica, Bosnia-Herzegovina, Brasil, Costa Rica, Dinamarca, Estonia, Finlandia, Francia, Irán, Irak, Japón, Kenia, México, Irlanda del Norte, Perú, Portugal, Escocia, Suiza, Taiwán o Tanzania.

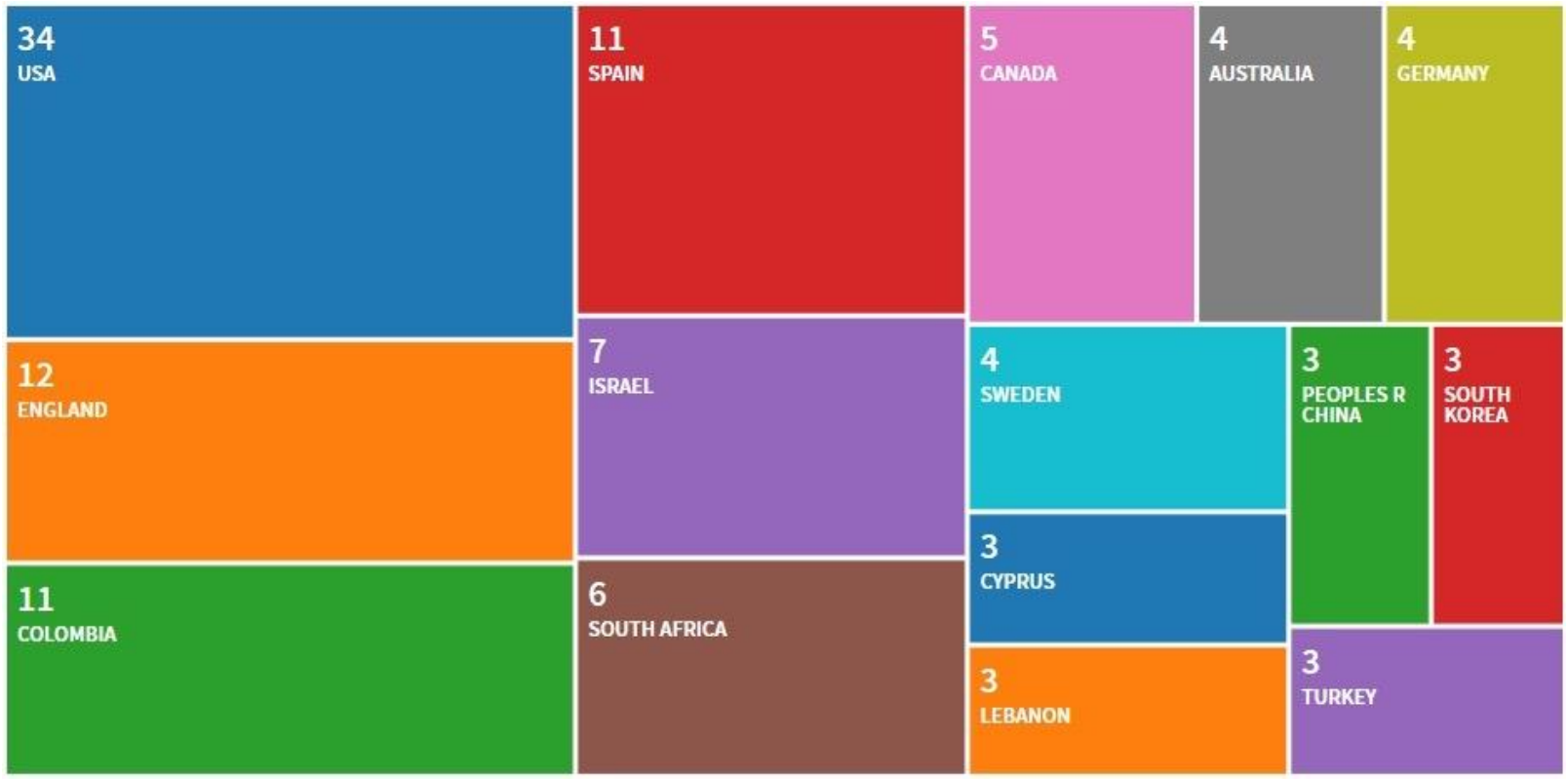

Figura 1. Principales frecuencias de publicación según los países a los que se adscriben los/las autores/as. Fuente: WOS.

En referencia al análisis interpretativo de los títulos, palabras clave y abstracts a través de las categorías establecidas, si comenzamos por el análisis del ámbito curricular desde el que se abordan los conflictos, vemos que la educación para la ciudadanía es el principal. En 42 publicaciones $(32,8 \%)$ se hace referencia exclusiva a esta área, mientras que el $50 \%(n=64)$ de ellas lo hace tanto desde la didáctica de las ciencias sociales como desde la educación para la ciudadanía. Por último, un $17,2 \%(n=22)$ se enmarca únicamente en la didáctica de las ciencias sociales. 
Respecto a la naturaleza de los trabajos, si bien con la información que ofrece el resumen de las publicaciones ésta no siempre se puede conocer con exactitud, en 108 de los casos (84,4\%) puede identificarse con seguridad que se trata de investigaciones, en la mayoría de los casos de carácter cualitativo. El resto, no ofrece información suficiente como para poder conocer si se ha realizado una investigación o no. Algunas de las publicaciones parecen tratarse de revisiones bibliográficas - ensayos, y otras describen propuestas educativas, de las cuales en 2 se dice que se han evaluado, pero sin ofrecer información suficiente como para ser consideradas investigación.

En cuanto a esas investigaciones identificadas, los trabajos de corte cualitativo son predominantes. Entre los 108 casos, el 58,3\% (n=63) declara explícitamente que se trata de una investigación cualitativa, o se interpreta sin dificultad por el tipo de estrategias (estudio de casos; investigaciónacción, de tipo etnográfico) o técnicas de investigación declaradas (técnicas documentales, entrevistas, grupos de discusión). En 9 casos (7\%) se describe únicamente el uso de cuestionarios, por lo que han sido consideradas investigaciones de corte cuantitativo. El resto de las publicaciones $(n=36 ; 33,3 \%$ ) no ofrecen detalles que permitan categorizar el tipo de investigación desarrollada, aunque en 5 casos podría tratarse de investigaciones de carácter mixto, ya que se citan técnicas tanto cualitativas como cuantitativas.

De la muestra total, el $66,4 \%(n=85)$ hace alusión a algún tipo de propuesta educativa (Tabla 7). Entre ellos, hay una mayor presencia de proyectos o experiencias educativas como intervenciones en aula, visitas a memoriales, aplicación de metodologías innovadoras y otro tipo de propuestas. También se ha escrito en torno a materiales educativos, siendo el análisis de libros de texto un tema recurrente en la investigación $(n=15)$, si bien pueden también encontrarse propuestas para la utilización de serious games, unidades didácticas, películas o revistas, entre otras. En tercer lugar, se encuentran una serie de programas educativos específicos, impulsados por universidades, escuelas, instituciones, asociaciones y museos y que, por lo general, parten de objetivos preventivos o de pacificación. Las políticas educativas impulsadas por los gobiernos ocupan el cuarto lugar, siendo en su seno el análisis curricular $(n=6)$ el ámbito que despierta un mayor interés. Finalmente, encontramos una serie de trabajos que realizan 0 analizan distintos tipos de propuestas al mismo tiempo, muchos en relación al curriculum y los libros de texto.

Tabla 7

Frecuencias para el tipo de propuesta educativa a la que se hace alusión

\begin{tabular}{lll}
\hline & $\mathrm{N}$ & $\%$ \\
\hline Proyecto/experiencia educativa & 31 & 36,5 \\
Material educativo & 20 & 23,6 \\
Programa educativo & 17 & 20 \\
Política educativa & 10 & 11,7 \\
Varios tipos de propuestas & 7 & 8,2 \\
Total & 85 & 100 \\
\hline
\end{tabular}

Fuente: elaboración propia. 
Existe entre los estudios cierta tendencia a realizar revisiones de la historia oficial enseñada, sea mediante el análisis del currículo oficial o de las narrativas de los libros de texto. En las investigaciones desarrolladas se detecta un llamamiento a la reflexión en torno a la repercusión que tienen los discursos planteados en la formación de la ciudadanía. Igualmente, se evidencia una crítica al tipo de discurso que se ha venido ofreciendo, detectando una serie de limitaciones de cara a una futura convivencia en paz y bajo el marco de los derechos humanos. En concreto, varios estudios concluyen que la educación para la ciudadanía se ha utilizado para unificar a la población alrededor de un régimen o una nación. Como caso contrario, el artículo 101 (Anexo I) señala a Sudáfrica como un referente a seguir en estas cuestiones. De manera paralela, podemos encontrar algunas críticas a programas actuales, como los impulsados en el Reino Unido de cara a combatir el terrorismo.

En cuanto a los contextos de enseñanza-aprendizaje, un $84,4 \%(n=108)$ de la muestra puede vincularse a la educación formal, bien porque las investigaciones se refieren a este contexto, bien porque presentan alguna propuesta o experiencia desarrollada en este ámbito. En 58 casos se puede conocer la etapa a la que se adscriben. Un $27,6 \%(n=16)$ de estos casos se sitúa en la multicategoría: en 1 se cita la educación básica y superior; y en 15 la educación básica, sin especificar más. Pero sabemos que los conflictos a los que nos referimos se trabajan principalmente en Educación Secundaria, que cuenta con un $46,6 \%(n=27)$ de casos de entre los 58 declarados, seguido de lejos por la Educación Superior $(n=9 ; 15,5 \%)$ y la Educación Primaria $(n=6 ; 10,3 \%)$.

Si atendemos al conflicto tratado, vemos que un 70,3\% $(n=90)$ de los estudios menciona algún conflicto armado de manera explícita. El restante $29,7 \%$ de los casos ( $n=38$ ) no explicitan el conflicto de modo directo, aunque se puede adivinar. En lo que se refiere al tipo de conflicto abordado, encontramos una casuística abundante (Tabla 8).

\section{Tabla 8}

Frecuencias para el tipo de conflicto armado

\begin{tabular}{lll}
\hline & $\mathrm{N}$ & $\%$ \\
\hline Guerra civil & 25 & 19,5 \\
Represión contra minorías & 25 & 19,5 \\
Grupos armados & 21 & 16,4 \\
Conflicto universal & 17 & 13,3 \\
Terrorismo yihadista & 13 & 10,2 \\
Conflictos entre países & 12 & 9,4 \\
Multicategoría & 9 & 7 \\
Conflicto colonial / post-colonial & 6 & 4,7 \\
Total & 128 & 100 \\
\hline
\end{tabular}

Fuente: elaboración propia. 
Hay una serie de estudios proyectados sobre varios conflictos a la vez o que hacen referencia a los conflictos y guerras a modo global. Entre los que se presentan de manera diferenciada, las guerras civiles son el tipo de conflicto más citado: guerras del Líbano, Guerra de los Balcanes, diferentes guerras civiles en África, de Guatemala, Sri Lanka, España, etc. También constituyen un número importante los casos en los que se trata la represión contra minorías. Aparecen en el seno de esta tipología conflictos asociados a minorías nacionales, como la kurda; a grupos raciales, como el caso del movimiento por los derechos civiles de Estados Unidos o el Apartheid de Sudáfrica; y también casos de violencia de Estado contra minorías ideológicas, como la derivada de las dictaduras de Brasil, Chile o Argentina. A los dos tipos anteriores, les siguen los conflictos en los que intervienen grupos armados. El protagonismo en esta tipología es acaparado por el conflicto colombiano, aunque también están presentes otras vivencias marcadas por el terrorismo en Irlanda del Norte, País Vasco o Perú. Por su parte, entre los conflictos entre diferentes naciones, el más reseñable es el árabe-israelí. En menor medida se hallan los conflictos coloniales o post-coloniales de Papúa Nueva Guinea, algunas regiones de China o países de América.

Pero además de los conflictos localizados en un territorio concreto, también están presentes los conflictos armados que pueden ser considerados de carácter universal, como son las dos guerras mundiales y la Guerra Fría; o el terrorismo yihadista. En el caso de la Guerra Fría y las guerras mundiales, además de presentar propuestas para trabajarlas en el aula $(n=6)$, muchos trabajos se centran en analizar cómo han sido representadas en la enseñanza de la historia $(n=11)$, mostrando en algún caso la repercusión que tuvieron en la formación ciudadana o enseñanza de la historia. Entre las publicaciones referidas al terrorismo yihadista destacan las 5 aportaciones que analizan programas preventivos llevados a cabo en el Reino Unido.

Un $20,3 \%(n=26)$ de los casos no se pueden relacionar con un lugar concreto: bien porque se tratan de manera generalizada sin especificar ningún conflicto en concreto; por tratarse de conflictos de carácter universal; o porque son casos recogidos como multicategoría, entre los que se citan lugares como Alemania, País Vasco, Irlanda, Argentina o Vietnam. De entre el resto (Tabla 9), el conflicto colombiano copa gran parte de las publicaciones, aunque también tienen una presencia notable los conflictos que repercuten a Estados Unidos (sus guerras civiles, el racismo o el terrorismo yihadista). En menor medida, se hallan el Apartheid en Sudáfrica, el terrorismo en Reino Unido, el conflicto de Oriente Medio o las guerras civiles del Líbano.

\section{Tabla 9}

Frecuencias para los territorios en los que se dan los conflictos

\begin{tabular}{lll}
\hline$N$ & $\%$ & Territorios del conflicto \\
\hline 14 & 10,9 & Colombia \\
10 & 7,8 & Estados Unidos \\
6 & 4,7 & Israel y Sudáfrica \\
5 & 3,9 & Reino Unido \\
4 & 3,1 & Líbano \\
3 & 2,3 & Chipre, España, Guatemala, Irlanda y Pakistán
\end{tabular}


2 1,6 Canadá, Chile, China, Holanda, Indonesia, Kosovo, Liberia y Turquía

$1 \quad$ 0,8 Alemania, Australia, Bosnia, Brasil, Corea, Costa de Marfil, Estonia, Finlandia, Francia, Irak, Italia, Kenia, Kurdistán, Laos, Nueva Zelanda, Papúa Nueva Guinea, Perú, Polonia, Sri Lanka, Sudán, Sudán del Sur, Suiza, Taiwán, Tanzania, Uganda y Vietnam

Fuente: elaboración propia.

En lo que respecta a la datación de los conflictos (Tabla 10), predomina con el $84,4 \%(n=108)$, los que aún cuentan con supervivientes, frente a los que sucedieron hace más de 100 años (Conquista de América, I Guerra Mundial y guerras civiles estadounidenses). Entre los primeros, destacan los conflictos que siguen hoy en día vigentes, como el terrorismo yihadista o el conflicto árabe-israelí; seguidos de los que han finalizado en los últimos 25 años, caso de Colombia. Tras ellos, figuran los conflictos que finalizaron entre hace 25 y 50 años, entre los que encontramos el Apartheid sudafricano o la Guerra Fría. Por último, se localizan los que terminaron entre hace 50 y 100 años, con gran repercusión de la ll Guerra mundial y el Holocausto. Existe además un grupo de estudios clasificados como multicategoría que no puede encajarse en esta gradación, porque en su seno se abordan distintos conflictos de diferentes periodos. 


\section{Tabla 10}

Frecuencias para los periodos de finalización de los conflictos

\begin{tabular}{lllc}
\hline & & $N$ & $\%$ \\
\hline Anterior a 1920 & Hace más de 100 años (antes de 1919) & 10 & 7,8 \\
Posterior a 1920 & Hace entre 100-50 años (1920-1969) & 17 & 13,3 \\
& Hace entre 50-25 años (1970-1994) & 18 & 14,1 \\
& Últimos 25 años (entre 1995-2020) & 36 & 28,1 \\
& Vigente & 37 & 28,9 \\
Multicategoría & & 10 & 7,8 \\
\hline Total & & 128 & 100 \\
\hline
\end{tabular}

Fuente: elaboración propia.

La mayoría de publicaciones hacen referencia a cuestiones referidas al conflicto en sí, frente a aquellos que hacen referencia a términos educativos vinculados a la paz y la convivencia (Tabla 11). Guerra es el término de búsqueda más utilizado, seguido de violencia aunque, en este último caso, no siempre se hace referencia a violencia armada. Por el contrario, el término conflicto armado solo se utiliza para hacer referencia a lo sucedido en el Líbano $(n=2)$ y en Colombia $(n=6)$.

El término terrorismo, es algo menos utilizado. Se encuentra también como contraterrorismo $(n=6)$ y se asocia al radicalismo y el extremismo $(n=7)$. En los casos en los que la información existente permite identificar el tipo de terrorismo, siempre se refiere al yihadista $(n=7)$ y, la mayoría de propuestas $(n=7)$, se abordan desde la prevención del mismo.

Por su parte, salvo en el caso de las publicaciones sobre Alemania $(n=1)$, Sudáfrica $(n=1)$ y Bali $(n=1)$, el término víctimas se utiliza en contextos de habla hispana: Colombia $(n=3)$, Chile $(n=2)$ y España $(n=2)$. En Sudáfrica se refiere a las víctimas del Apartheid y en la publicación referida a Bali se asocia a un acto terrorista. En el caso del País Vasco, si bien no se cita, sabemos que también ha existido el terrorismo. El resto de publicaciones en las que aparece se encuadran en contextos donde se ha vivido una dictadura. Un último caso no especifica ningún conflicto concreto.

El término tortura no se ha encontrado, al igual que educación para el desarme.

Educación para la paz es algo más citado que educación para los derechos humanos. En ambos casos, no son pocos los casos en los que las publicaciones se han localizado exclusivamente a través de educación para la paz $(\mathrm{n}=10)$ o educación para los derechos humanos $(\mathrm{n}=9)$, sin referencias a ningún otro término de búsqueda. Por otro lado, aunque ambos se emplean para referirse a contextos diversos, la educación para la paz es citada en 5 trabajos que abordan la cuestión colombiana y en otros 3 referidos a Israel. 


\section{Tabla 11}

$N .^{\circ}$ de publicaciones que contienen los términos de búsqueda referidos a la temática a tratar

\begin{tabular}{|c|c|c|c|}
\hline Término de búsqueda & $\mathrm{N}$ & $\%$ & Artículos (listado en anexol) \\
\hline Terrorism & 12 & 9,4 & $5,15,27,57,67,74,79,94,107,115,118,122$ \\
\hline Torture & 0 & 0 & \\
\hline Violence & 36 & 28,1 & $\begin{array}{l}10,11-16,19,22,24,28-31,34,37,38,40,42, \\
43,45,47,48,56,58,61,66,80,83,84,97,99, \\
102,111,114,124\end{array}$ \\
\hline War & 60 & 46,88 & $\begin{array}{l}4,6,7,10-13,17,20,21,23,26,28,33,39,41, \\
44,45,47,48,51,53-55,58,59,62-65,68,70, \\
72,73,75,78,80,85-87,89,90,93,95,96,100, \\
103,105,106,108,109,112,113,117,119-121, \\
125-127\end{array}$ \\
\hline Armed conflict & 8 & 6,25 & $1-3,43,69,81,85,98$ \\
\hline Victims & 11 & 8,6 & $33,40,42,43,71,77,81,92,110,115,116$ \\
\hline Peace education & 15 & 11,7 & $\begin{array}{l}13,16,18,25,28,32,36,39,49,50,52,60,88, \\
91,115\end{array}$ \\
\hline Disarmament education & 0 & 0 & \\
\hline Human rights education & 12 & 9,4 & $8,9,35,46,47,76,82,100,101,104,123,128$ \\
\hline
\end{tabular}

Fuente: elaboración propia.

En cuanto al informe de citas que ofrece WOS, vemos que, a nivel global, entre las 128 publicaciones que conforman la muestra, se obtiene un índice h de 14. Entre todos los trabajos, se han recibido 583 citas en la colección principal de WOS (535 eliminando autocitas), realizadas en 503 trabajos, 475 de los cuales no forman parte de la muestra. 57 (44,5\%) de las publicaciones no han sido citadas, y $32(25 \%)$ no sobrepasan las 2 menciones. Por tanto, vemos que la mayoría de citas recibidas se concentran en las $12(9,3 \%)$ publicaciones que ostentan más de 15 (Publicaciones 11, 19, 49,71, 85, 88, 91, 94, 113, 114,118 y 125), especialmente, entre los artículos 19 (71 citas) y 125 (68 citas), escritos respectivamente por Brown y Brown (2010) y Wineburg, Mosborg, Porat y Duncan (2007). Los otros 10 trabajos no superan las 25 citas.

En cuanto al acceso, solo el $26,6 \%(n=34)$ de los trabajos cuenta con acceso libre, sin existir relación con el número de citas recibidas, puesto que entre los 12 trabajos con más referencias solamente 2 de ellos se encuentran bajo acceso libre y no son los que más citas reciben.

Tampoco la antigüedad de los trabajos se relaciona con las citas recibidas, dado que 6 de los artículos más citados fueron publicados entre los años 2014 y 2016 y, los restantes 6 entre 2008 y 2010. En los últimos tres años, no existen publicaciones con más de 15 citas, pero, por el contrario, resulta representativo que la mayoría de citas $(n=391)$ se reciban en publicaciones que han visto la luz a partir de 2017 (Figura 2), momento en el que comienza a publicarse de manera más notable sobre el tema (Figura 3 ). 


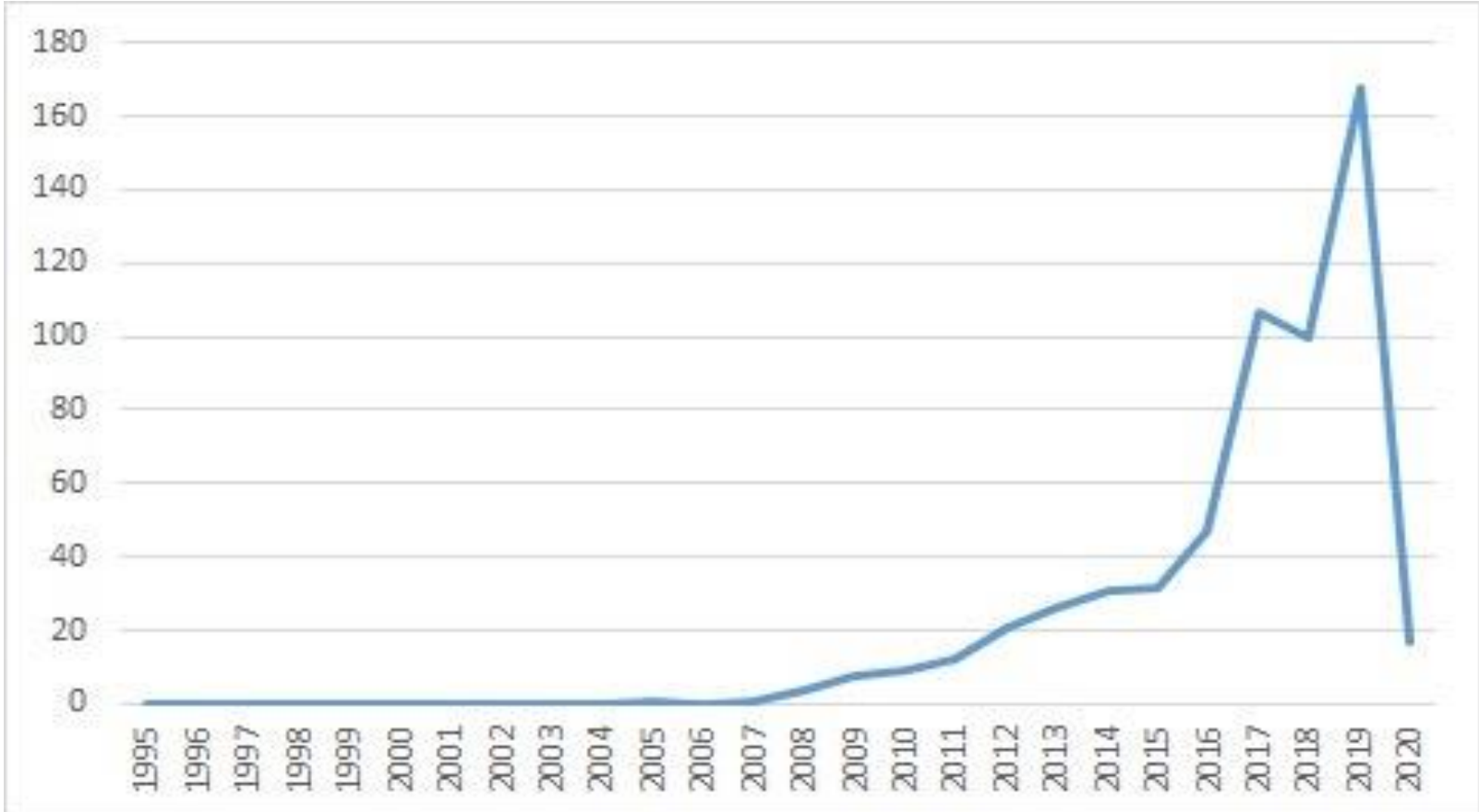

Figura 2. Citas recibidas cada año por la muestra. Fuente: elaboración propia a partir de los datos de WOS.

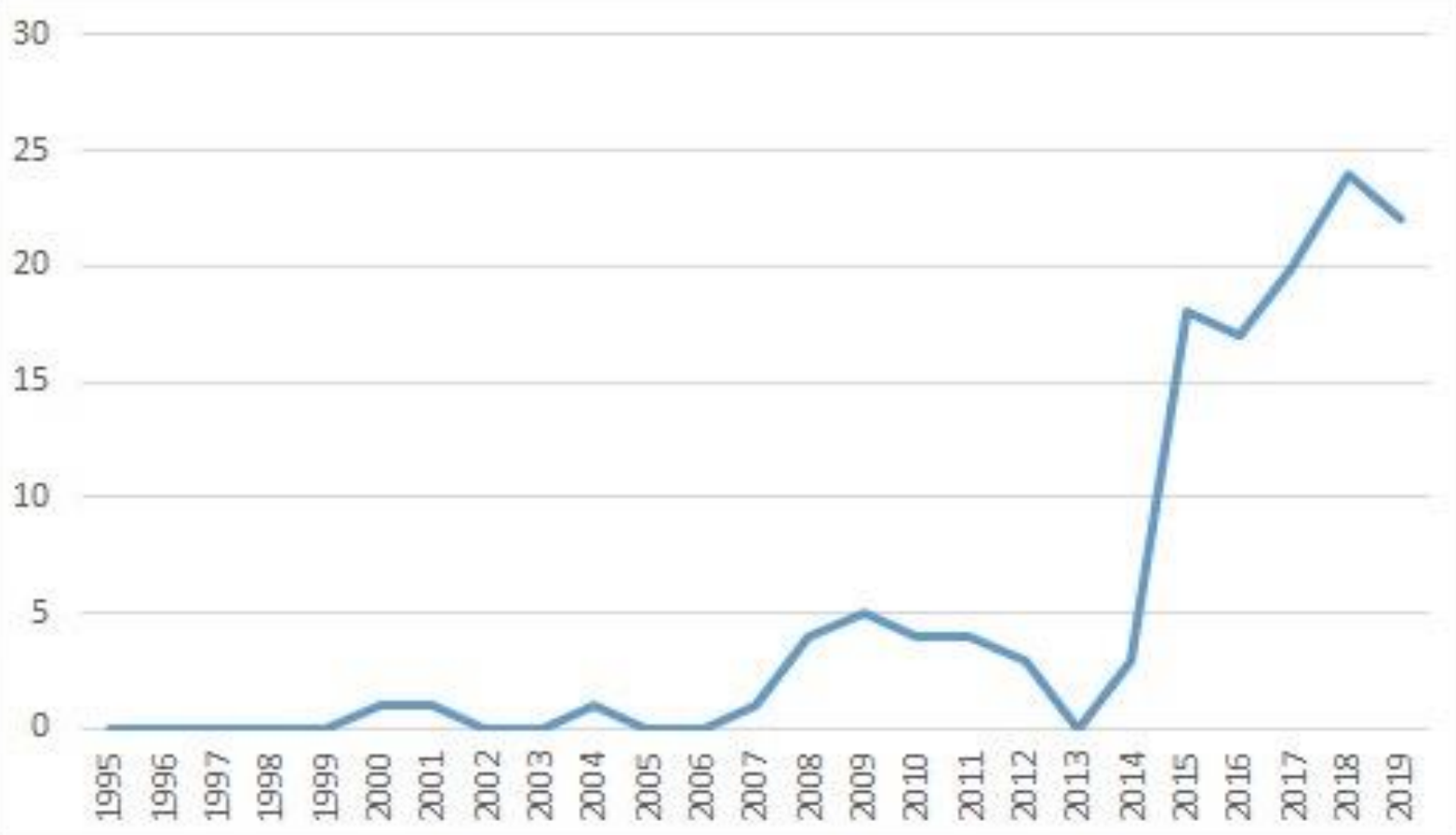

Figura 3. Años de publicación de los trabajos de la muestra. Fuente: elaboración propia a partir de los datos de WOS.

\section{Desarrollo y discusión}

Como señalan diversos/as autores/as (Rubin y Cervinkova, 2019; Toledo Jofré et al., 2015) el tratamiento de temas controvertidos desde un punto de vista educativo no resulta sencillo. Probablemente por eso el, a nuestro entender, reducido número de publicaciones que conforman la muestra, indica que se trata de un tema poco tratado en la literatura científica, aunque se infiere que desde el año 2015 va tomando mayor fuerza, en consonancia con las reivindicaciones 
que desde la didáctica de las ciencias sociales se están haciendo para empezar a trabajar estas cuestiones (Jiménez Martínez y Felices de la Fuente, 2018; López Facal y Santidrián, 2011). Probablemente también tenga algo que ver: por un lado, la cada vez mayor exigencia, también en el área de didáctica de las ciencias sociales, de publicar en bases de datos como WOS; y por otro, como ha ocurrido en otras subáreas, la incorporación a WOS Core Collection de Emerging Science Citation Index-ESCl (Fontal e lbañez-Etxeberria, 2017). A los citados factores se asocia el que la mayoría de citas detectadas en la muestra se hayan realizado a partir de 2017, ya que, cuanto más se escribe sobre el tema, más referencias se realizan.

De todas formas, el índice h (14) de la muestra es moderado, y son pocos los trabajos que cuentan con un número reseñable de citas, existiendo únicamente dos artículos que pueden ser considerados referentes; Brown y Brown (2010) y Wineburg et al. (2007). También, las autocitas dentro de la muestra son escasas. Del mismo modo, no se identifican autores/as o equipos que trabajen de manera sistematizada sobre el tema. Esto lleva a pensar que, cuando se abordan los conflictos armados de motivación política de larga duración desde la didáctica de las ciencias sociales o educación para la ciudadanía, generalmente se hace de manera cuasi-personal y aislada, sin engarzarlos suficientemente con trabajos previos que puedan ser de utilidad, y sin responder a programas de investigación a medio-largo plazo. Aun así, a lo largo de las diferentes fases de cribado, se han localizado una serie de autores/as que han trabajado cuestiones en torno a la educación para la ciudadanía (Barton, 2015; Bickmore, Kaderi y GuerraSua, 2017; Finkel, Horowitz y Rojo-Mendoza, 2012; Nygren y Jonhsrud, 2018; Rubin, 2016; Sen, 2019), que se encuentran también en la muestra desarrollando experiencias concretas vinculadas a los conflictos armados políticos de larga duración.

Si bien, la falta de publicaciones en plataformas de acceso libre podría suponer un problema a la hora de acceder a los trabajos ya existentes, no parece que está sea la razón para que no se tengan en cuenta, ya que los datos no indican que exista relación entre estas dos variables. En cualquier caso, de cara a futuro, parece interesante considerar la publicación en revistas de acceso libre, en cuanto que se estaría aportando información interesante de cara a la mejora de la convivencia en distintas sociedades. Si hablamos de educación para la ciudadanía y ésta reivindica la participación ciudadana, quizás deberíamos de predicar con el ejemplo y ofrecer a la sociedad nuestros estudios, así como intentar trabajar más en red.

Al igual que se ha visto en otros estudios bibliométricos vinculados a la didáctica de las ciencias sociales (Gómez-Carrasco, López-Facal y Rodríguez-Medina, 2019), el tipo de trabajos publicados constituyen en su gran mayoría investigaciones, especialmente asociadas a la evaluación de proyectos o intervenciones didácticas de distinto tipo. Un dato nada extraño si consideramos que se ha trabajado con una base de datos especializada en investigación. Sin embargo, llama más la atención que en WOS predomine la investigación de corte cualitativo (Bellino, 2016; Bermúdez y Argumero, 2018; Seixas, Peck y Poyntz, 2011 ; Stoddard, 2009), lo que seguramente sea un síntoma de la necesidad de realizar este tipo de investigaciones en el campo educativo, así como de su cada vez mayor reconocimiento.

La vinculación de los trabajos al campo de la educación es clara, tanto por su enfoque como por las revistas elegidas para su publicación. A grandes rasgos, su proyección se limita a la educación formal (Arias Ferrer, Egea Vivancos, Sánchez lbáñez, Domínguez Castillo, García Crespo y Miralles Martínez, 2019; Brown y Brown, 2016; del Pozo Serrano, 2016; Waldron y Oberman, 
2016), pero podría ser interesante ampliarla a otros ámbitos informales, como los memoriales u otros espacios de presentación del patrimonio. No en vano, este tipo de lugares ofrecen un componente emocional interesante para trabajar temáticas conflictivas y traumáticas. Paralelamente, la mayoría de los estudios analizados se asocian a la educación básica, en tanto que es la encargada de impulsar la competencia cívica (Eurydice, 2012). Es la etapa de secundaria la que mayor presencia tiene en la literatura, probablemente, porque los temas tratados requieren de cierta madurez para su comprensión. Aun así, consideramos que pude ser interesante trabajarlos desde etapas más tempranas, sobre todo desde un punto de vista emocional y en lugares donde el conflicto está aún vigente. Aprender a lidiar con los conflictos desde pequeños/as puede favorecer las actitudes de respeto hacia los demás, así como la interpretación crítica de la realidad.

La educación para la ciudadanía es el ámbito curricular desde el que se abordan los conflictos armados de carácter político de larga duración, pero la didáctica de las ciencias sociales y, en concreto de la historia, está también muy presente, lo cual no sorprende en cuanto que la ciudadanía y valores éticos son uno de los campos de investigación identificados en la didáctica de las ciencias sociales (Gómez-Carrasco, López-Facal y Rodríguez-Medina, 2019). Durante la fase de cribado de la muestra, se ha constatado que una de las consecuencias de los conflictos armados -caso, por ejemplo, de las guerras mundiales-, es que suelen suponer un hito temporal a partir del cual la educación para la ciudadanía y la enseñanza de las ciencias sociales son reformuladas. Ambos campos educativos son delicados y exigen prestar atención al modo en el que se abordan en las aulas. De hecho, varias publicaciones (Darrow, 2008; Sen, 2019) analizan el modo en el que se han tratado dichos campos después de algún conflicto, identificando una serie de limitaciones y concluyendo que pueden ser mal utilizados para unificar a la población en torno a un régimen o una nación. Pese a ello, no podemos dejar de pensar que estos ámbitos educativos constituyen una oportunidad para abordar cuestiones controvertidas (Santisteban Fernández, 2019), en busca de la paz y la convivencia desde el respeto a los derechos humanos, también en el País Vasco (Usón González, 2017). Como señala Banks (2017), este debe de ser un objetivo primordial de la didáctica de las ciencias sociales y, las investigaciones que se están desarrollando, deben seguir dando respuestas entorno al potencial de la educación para la ciudadanía como recurso para asentar la convivencia pacífica en nuestras sociedades democráticas.

En la muestra, el tipo de conflictos con mayor peso son las guerras civiles y la represión contra minorías, seguramente por ser urgente la reconciliación social en dichos lugares, puesto que restaurar la convivencia entre los grupos enfrentados en el pasado es allí más necesario que en otros lugares. Para la primera tipología de conflicto, encontramos abundantes países africanos (Davids y Waghid, 2016; Finkel, Horowitz y Rojo-Mendoza, 2012; Mvukiyehe y Samii, 2017; Skaras, 2019), de los Balcanes (Emkic, 2018), Líbano (Akar y Albrecht, 2017; Baytiyeh, 2017) o Guatemala (Bellino, 2016; Rubin, 2016). La segunda aúna en su seno a varios casos relativos al tratamiento del Apartheid sudafricano (Davids y Waghid, 2016; Russell, Sirota y Ahmed, 2019; Teeger, 2015), el caso chipriota (Zembylas, Charalambous, Charalambous y Lesta, 2016), el paquistaní (Emerson, 2018a y b, Kadiwal y Durrani, 2018), el kurdo (Darweish y Mohammed, 2018) o la minoría afroamericana estadounidense (Brown y Brown, 2010; Nygren y Johnsrud, 2018; Wills, 2019). En el otro extremo, destaca la escasez del tratamiento de conflictos coloniales o postcoloniales (Bermúdez y Argumero, 2018; Keynes, 2019; Tse, 2001), que parecen ser temas 
más difíciles de abordar, o quizás también por tratarse de cuestiones que vienen de lejos y estén, en cierta manera, asimiladas.

Es llamativo que los conflictos que más se tratan son aquellos que resultan más cercanos en el tiempo, ya que más de la mitad de los casos se centran en conflictos que, o bien siguen vigentes, - bien han finalizado en los últimos 25 años. Un hecho que volvería a asociarse a la necesidad de utilizar la educación para la ciudadanía como un instrumento de gran utilidad para restablecer la convivencia y el entendimiento entre grupos sociales enfrentados. Pero, antes de que estalle el conflicto armado, también puede utilizarse de manera preventiva, como se ha pretendido en Reino Unido con el terrorismo -aunque los resultados no parecen ser los más deseables (Mcghee y Zhang, 2017; Richardson, 2015; Thomas, 2016)-. De hecho, en un momento en el que los discursos de odio parecen extenderse, los temas controvertidos deben de tratarse en el aula.

En cuanto al aspecto geográfico, destaca de manera muy notable el gran peso de las investigaciones realizadas en Colombia, entre las que destacan las de Carrera-Díaz (2018) y Rodríguez, Bautista y Ramírez (2015). No en vano, se trata de un país que está actualmente inmerso en un proceso de reconciliación social y que cuenta con una amplia red de agentes, tanto estatales como asociativos, que promocionan con dinamismo variadas iniciativas en dicha dirección desde el campo de la educación. En contraposición, encontramos lugares como el País Vasco sobre el que solamente se ha localizado el artículo de Etxeberria-Mauleon (2018).

Si nos centramos en la metodología que hemos utilizado para la realización de este estudio, concluimos que el idioma utilizado en las búsquedas es el adecuado, pues la mayoría se ha escrito en inglés y el resto presenta el abstract y las palabras clave en ese idioma. Eso permite partir de los tesauros para la elección de los términos de búsqueda, en tanto que unifica los criterios empleados por el personal investigador, aunque ofrece algún error. Es el caso de social science education, el cual se emplea en plural -social sciences education-. Aun así, si bien se ha identificado algún término no recogido, como history teaching, estos han sido casos aislados. Por lo general, consideramos que los términos utilizados han dado buenos resultados, aunque pueden mejorarse.

En cuanto a los términos, parece significativo no haber encontrado ninguna referencia a la tortura. Un dato que relacionamos, partiendo de la idea de que la tortura suele estar muchas veces vinculada a la violencia de Estado silenciada, a dos posibles factores: la falta de datos al respecto o el temor a revisar la historia oficial del lugar en cuestión. En la muestra existe alguna referencia a las dictaduras de España (Delgado Algarra y Estepa Giménez, 2018), Argentina (Suárez, 2008) o Chile (Reyes, Cruz y Aguirre, 2016), de donde colegimos que podría ser interesante incluir el concepto dictadura en próximas búsquedas, de cara a localizar más publicaciones que traten la violencia de Estado. Por otro lado, consideramos que el término violencia debería de afinarse más, al igual que se ha hecho con conflicto al utilizar conflicto armado. Algunos de los términos identificados y susceptibles de utilizarse en un futuro son political violence, state violence o racial violence, todas ellas empleadas entre las publicaciones de la muestra.

Por otro lado, nos asaltan dudas respecto al empleo de los términos utilizados vinculados a los procesos educativos de pacificación. Por una parte, disarmament education no ha ofrecido ningún resultado. Por otra, varias publicaciones recogidas a través de la educación para la paz y educación para los derechos humanos han sido excluidas en la $3 .^{a}$ fase de cribado, por no 
corresponder a un contexto en el que existe un conflicto armado de motivación política alargado en el tiempo. Sin embargo, son varios los casos localizados a través del uso exclusivo de estos términos. Por lo tanto, a pesar de que muchas de las publicaciones halladas haciendo uso de estos términos de búsqueda sean susceptibles de ser eliminadas bajo los criterios de exclusión, creemos que en futuros trabajos deberían incluirse. Incluso podrían añadirse otros términos similares como peacemaking o peacebuilding, dando pie a la localización de un mayor número de experiencias.

Por último, consideramos que este estudio debería de completarse con los resultados obtenidos a través de la base de datos Scopus, en tanto que recoge gran cantidad de publicaciones del área (Fontal e Ibañez-Etxeberria, 2017), destacando un mayor peso entre los artículos españoles referidos a la ciudadanía y valores éticos (Gómez-Carrasco, López-Facal y Rodríguez-Medina, 2019).

\section{Conclusiones y limitaciones del estudio}

El análisis del tratamiento de temas socialmente controvertidos en educación constituye actualmente un campo de presencia aún moderada, pero con una tendencia progresiva al alza. Nuestro estudio ha revelado un variado y poco estructurado panorama internacional, muy amplio en cuanto a países y a enfoques desde los que se abordan escenarios de violencia o postviolencia. No en vano, en la muestra han hecho aparición más de 45 países o regiones afectadas por conflictos de variada naturaleza. Al respecto, Colombia se erige como un referente, dado que es un país en el que llevan varios años desarrollando experiencias y realizando una amplia labor investigadora. Ha quedado patente el mayor interés por abordar aquellos conflictos que aun cuentan con supervivientes $y$, en especial, los que siguen vigentes o son muy recientes. Así, podemos concluir que trabajar en el aula cuestiones conflictivas cercanas en el tiempo, en las que aún existe debate social, constituye un ámbito respecto al cual la educación tiene una gran responsabilidad, de cara a promover procesos de pacificación o reconciliación.

De ahí que pensemos que, también en el País Vasco, se debe abordar en las aulas el conflicto vivido en las décadas pasadas. Todavía es un tema incipiente y poco trabajado, pero esto no debe suponer un impedimento a la hora de tratarlo (Aranguren e lbañez-Etxeberria, 2020). El programa Adi-adian, a través de la transmisión que ofrece de las memorias asociadas al conflicto vivido, puede resultar una vía enriquecedora de cara a la reconciliación social. Así, consideramos especialmente importante seguir trabajando específicamente con el futuro profesorado para que aprendan a abordar los temas controvertidos en el aula, perdiendo el miedo al papel de acompañante en los debates a promover. En paralelo, parece pertinente sugerir la superación del vacío que el tratamiento de estas cuestiones presenta para las etapas de educación infantil y primaria. Por otro lado, las limitaciones que en nuestro estudio han aflorado sobre el planteamiento que en ocasiones se hace de la enseñanza de la competencia ciudadana, nos llevan a plantear la necesidad de desarrollar investigaciones educativas, evaluaciones de experiencias y programas, como en el caso que nos ocupa.

Para terminar, entendemos que nuestra revisión tiene ciertas limitaciones. Por un lado, porque únicamente se han tenido en cuenta los títulos, palabras clave y resúmenes de las publicaciones, lo cual reduce la información y las posibilidades de interpretación. Por otro, porque la búsqueda se ha realizado exclusivamente a través de la base de datos WOS. Por ello, planteamos como 
futuros pasos: la lectura de las publicaciones identificadas en la muestra, así como su ampliación a través de la incorporación de nuevas referencias localizadas en otras bases de datos. Una de ellas debería ser Scopus, pues representa la segunda base de datos de referencia para el campo educativo y con gran peso en la didáctica de las ciencias sociales. Para el caso concreto de España y otros países de habla hispana, podría incorporarse también Dialnet.

\section{Agradecimientos}

Este proyecto ha contado con financiación del Vicerrectorado de Innovación, Compromiso Social y Acción Cultural de la Universidad del País Vasco (UPV-EHU) a través del Proyecto de Innovación Educativa Memoria y convivencia (HBP2019-20/102); y del Gobierno Vasco a través del Grupo de Investigación en Arqueología Medieval, Patrimonialización y Paisajes Culturales/Erdi Aroko Arkeologia, Ondaregintza eta Kultur Paisaiak Ikerketa Taldea de la UPV/EHU (IT1 193-19) y del Grupo de Investigación Sociedad Poder y Cultura (XIV-XVIII) (IT-819-16).

\section{Bibliografía}

Akar, B. (2016). Dialogic pedagogies in educational settings for active citizenship, social cohesion and peacebuilding in Lebanon. Education Citizenship and Social Justice, 11 (1), 44-62.

Akar, B., y Albrecht, M. (2017). Influences of nationalisms on citizenship education: revealing a "dark side' in Lebanon. Nations and Nationalism, 23(3), 547-570.

Albas, L., Ibañez-Etxeberria, A., Echeberria, B. y Vicent, N. (en prensa). El tratamiento de la violencia en las aulas en el País Vasco. Análisis exploratorio de la implementación del módulo educativo Adi-Adian en la formación inicial del profesorado de Educación Infantil.

Aranguren, O. e Ibañez-Etxeberria, A. (2020). Euskal kasuari lotutako motibazio politikoko indarkeriaren trataera Hezkuntzan: Adi-Adian Hezkuntza Modulua Haur Hezkuntza Graduko irakaslegaiengan. En Gamito Gómez, R., Martínez Abajo, J., y Vizcarra Morales, M. T. (Eds.), XXVI Jornadas de Investigación en Psicodidáctica (pp. 219-231). Leioa: Universidad del País Vasco.

Arias Ferrer, L., Egea Vivancos, A., Sánchez lbáñez, R., Domínguez Castillo, J., García Crespo, F. J., y Miralles Martínez, P. (2019). Forgotten history or history not taught? The students of Spanish High School and their lack of knowledge about the Civil War. Revista Complutense de Educacion, 30(2), 461-478. doi: 10.5209/RCED.57625

Baliqi, B. (2017). The Aftermath of War Experiences on Kosovo's Generation on the Move Collective Memory and Ethnic Relations among Young Adults in Kosovo. Zeitgeschichte, 44(1), 6-19.

Baliqi, B. (2018). Contested war remembrance and ethnopolitical identities in Kosovo. Nationalities Papers-the Journal of Nationalism and Ethnicity, 46(3), 471-483.

Banks, J. A. (2017). Failed Citizenship and Transformative Civic Education. Educational Researcher, 46(7), 366-377. doi: $10.3102 / 0013189 \times 17726741$

Barton, K. C. (2015). Young adolescents' positioning of human rights: Findings from Colombia, Northern Ireland, Republic of Ireland and the United States. Research in Comparative and International Education, 10(1), 48-70. 
Baytiyeh, H. (2017). Has the Educational System in Lebanon Contributed to the Growing Sectarian Divisions?. Education and Urban Society, 49(5), 546-559.

Bellino, M. J. (2015). The Risks We Are Willing to Take: Youth Civic Development in "Postwar" Guatemala. Harvard Educational Review, 85(4), 537-561.

Bellino, M. J. (2016). So That We Do Not Fall Again History Education and Citizenship in "Postwar" Guatemala. Comparative Education Review, 60(1), 58-79.

Bermúdez, Á. y Martínez, D. A. (2018). The narrative framing of violence in teaching resources about the Spanish Conquest of America. Panta Rei: revista de ciencia y didáctica de la historia, 8, 93-118. doi: 10.6018/pantarei/2018/5

Bickmore, K., Kaderi, A. S., y Guerra-Sua, A. (2017). Creating capacities for peacebuilding citizenship: history and social studies curricula in Bangladesh, Canada, Colombia, and Mexico. Journal of Peace Education, $14(3), 282-309$.

Brown, A. L., y Brown, K. D. (2010). Strange Fruit Indeed: Interrogating Contemporary Textbook Representations of Racial Violence Toward African Americans. Teachers College Record, $112(1), 31-67$.

Calaf, R., Gutiérrez Berciano, S., y Suárez Suárez, M. Á. (2020). La evaluación en la Educación Patrimonial. 20 años de investigaciones y Congresos de ICOM. Aula Abierta, 49(1), 55-64. doi: $10.17811 /$ rifie.49.1.2020.55-64

Carrera-Díaz, P. E. (2018). Research for action, action for research with victims of armed conflict in Soacha, Colombia. Prospectiva, 25, 163-186.

Darrow, M. H. (2008). In the land of Joan of Arc: The civic education of girls and the prospect of war in France, 1871-1914. French Historical Studies, 31(2), 263-291.

Darweish, M., y Mohammed, M. A. (2018). History education in schools in Iraqi Kurdistan: representing values of peace and violence. Journal of Peace Education, 15(1), 48-75.

Davids, N., y Waghid, Y. (2015). On speaking to violence in post-apartheid schools. Tydskrif Vir Geesteswetenskappe, 55(4), 681-693.

Davids, N., y Waghid, Y. (2016). Higher education as a pedagogical site for citizenship education. Education Citizenship and Social Justice, 11(1), 34-43.

de Alba Fernández, N., García Pérez, F. F. y Santisteban Fernández, A. (Eds.) (201 2). Educar para la participación ciudadana en la enseñanza de las ciencias sociales. Sevilla: AUPDCS, Díada editora.

del Pozo Serrano, F. J. (2016). Social School Pedagogy in Colombia: the Model of Universidad Del Norte on School Management and Teaching Training for Citizenship and Peace. Revista Iberoamericana de Educación, 70, 77-90.

Delgado Algarra, E. J. y Estepa Giménez, J. (2018). Citizenship and dimensions of memory in the learning of history: analysis of a High School Education case. Vínculos de Historia, 7, 366388.

Dvir, Y., Yemini, M., Bronshtein, Y. y Natur, N. (2018). International education as a novel entity in a public education system: the establishment of a new public international school in Israel. Compare-a Journal of Comparative and International Education, 48(6), 935-953. 
Emerson, A. (2018). The making of the (II)legitimate citizen: the case of the Pakistan Studies textbook. Global Change Peace \& Security, 30(3), 295-311.

Emerson, A. (2018). Educating Pakistan's Daughters: Girls' Citizenship Education and the Reproduction of Cultural Violence in Pakistan. Studies in Social Justice, 12(2), 291-309.

Emkic, E. (2018). Peace Education in Bosnia and Herzegovina. Reconciliation and Education in Bosnia and Herzegovina: from Segregation to Sustainable Peace, 13, 37-49.

Etxeberria-Mauleon, X. (2018). The presentation of victims in civic education in situations of sociopolitical transition from violence to peace. Quaestiones Disputatae, $11(23), 189-217$.

Eurydice (2012). La educación para la ciudadanía en Europa. España: Ministerio De Educación, Cultura y Deporte.

Finkel, S. E., Horowitz, J. y Rojo-Mendoza, R. T. (2012). Civic Education and Democratic Backsliding in the Wake of Kenya's Post-2007 Election Violence. Journal of Politics, 74(1), 52-65.

Fontal, O. (2016). The Spanish Heritage Education Observatory. Cultura y Educación, 28(1), 254.

Fontal, O. e lbañez-Etxeberria, A. (2017). La investigación en Educación Patrimonial. Evolución y estado actual a través del análisis de indicadores de alto impacto. Revista de educación, $375,184-214$.

Gobierno Vasco (s.f.). Módulo educativo Adi-Adian. Aprendizajes de dignidad humana, convivencia y empatía mediante una experiencia de escucha de testimonios de víctimas. https://www.euskadi.eus/gobierno-vasco/-/modulo-adi-adian/

Gobierno Vasco (2008). Plan Vasco de Educación para la Paz y los Derechos Humanos (20082011 ).

Gobierno Vasco (2010). Plan de Convivencia Democrática y Deslegitimación de la violencia (20102011 ). Reformulación del Plan Vasco de Educación para la Paz y los DD.HH. (2008-201 1). https://www.euskadi.eus/gobierno-vasco/-/plan-gubernamental/2-convivenciademocratica-y-deslegitimacion-de-la-violencia-20102011/

Gobierno Vasco (2013). I Plan de Paz y Convivencia 2013-16. Un objetivo de encuentro social. https://www.euskadi.eus/documentos-paz-convivencia/web01-s1lehbak/es/

Gobierno Vasco (2017). Plan de Convivencia y Derechos Humanos 2017-2020. Un objetivo de encuentro social, la opción por la empatía. https://www.euskadi.eus/documentos-pazconvivencia/web01-s1lehbak/es/

Gómez-Carrasco, C. J., López-Facal, R. y Rodríguez-Medina, J. (2019). La investigación en Didáctica de las Ciencias Sociales en revistas españolas de Ciencias de la Educación. Un análisis bibliométrico (2007-2017). Didáctica de las Ciencias Experimentales y Sociales, 37, 67-88.

Goren, H. y Yemini, M. (2016). Global citizenship education in context: teacher perceptions at an international school and a local Israeli school. Compare-a Journal of Comparative and International Education, 46(5), 832-853.

Goren, H., Maxwell, C. y Yemini, M. (2019). Israeli teachers make sense of global citizenship education in a divided society- religion, marginalisation and economic globalisation. Comparative Education, 55(2), 243-263. 
Guerra-Sua, A. M. (2019). Challenges for Peacebuilding and Citizenship Learning in Colombia. Magis-Revista Internacional de Investigacion en Educacion, 11 (23), 169-186.

Jerome, L. y Elwick, A. (2019) Teaching about terrorism, extremism and radicalisation: some implications for controversial issues pedagogy. Oxford Review of Education.

Jiménez Martínez, M. D. y Felices de la Fuente, M. M. (2018). Cuestiones socialmente vivas en la formación inicial del profesorado: la infancia refugiada siria como problemática. REIDICS, 3, 87-102.

Keynes, M. (2019). History Education for Transitional Justice? Challenges, Limitations and Possibilities for Settler Colonial Australia. International Journal of Transitional Justice, 13(1), 113-133.

Lee, J., y Elwick, A. (2019). Identifying an Educational Response to the Prevent Policy: Student Perspectives on Learning about Terrorism, Extremism and Radicalisation. British Journal of Educational Studies, 67(1), 97-114.

López Facal, R. y Santiadrián, V. M. (2011). Los "conflictos sociales candentes" en el aula. Íber. Didáctica de las Ciencias Sociales, Geografía e Historia, 69, 8-20.

McGhee, D. y Zhang, S. (2017). Nurturing resilient future citizens through value consistency vs. the retreat from multiculturalism and securitisation in the promotion of British values in schools in the UK. Citizenship Studies, 21 (8), 937-950.

Mvukiyehe, E. y Samii, C. (2017). Promoting Democracy in Fragile States: Field Experimental Evidence from Liberia. World Development, 95, 254-267.

Nygren, T. y Johnsrud, B. (2018). What Would Martin Luther King Jr. Say? Teaching the Historical and Practical Past to Promote Human Rights in Education. Journal of Human Rights Practice, 10(2), 287-306.

Pineda-Alfonso, José A. (2015). Educar para la ciudadanía trabajando con temas controvertidos en Educación Secundaria Obligatoria. Revista de Investigación Educativa, 33(2), 353-367.

Philippou, S. (2009). What makes Cyprus European? Curricular responses of Greek-Cypriot civic education to 'Europe'. Journal of Curriculum Studies, $41(2), 199-223$.

Philippou, S. y Theodorou, E. (2019). Collapsing the Supranational and the National. From Citizenship to Health Education in the Republic of Cyprus. En A. Rapoport (ed.), Competing Frameworks. Global and National in Citizenship Education (pp. 95-114). USA: Information Age Publishing.

Reyes, M. J., Cruz, M. A. y Aguirre, F. J. (2016). Places of memory and the new generations: Some political effects of the transmission of memories of Chile's recent past. Revista Española de Ciencia Política-RECP, 41, 93-114.

Rubin, B. C. (2016). We Come to Form Ourselves Bit by Bit: Educating for Citizenship in PostConflict Guatemala. American Educational Research Journal, 53(3), 639-672.

Rubin, B. C. y Cervinkova, H. (2019). Challenging Silences: Democratic Citizenship Education and historical Memory in Poland and Guatemala. Anthropology \& Education Quarterly, 0(0), 1 17. 
Russell, S. G., Sirota, S. L. y Ahmed, A. K. (2019). Human Rights Education in South Africa: Ideological Shifts and Curricular Reforms. Comparative Education Review, 63(1), 1-27.

Santisteban Fernández, A. (2019). La enseñanza de las Ciencias Sociales a partir de problemas sociales o temas controvertidos: estado de la cuestión y resultados de una investigación. El Futuro del Pasado, 10, 57-79.

Seixas, P., Peck, C. y Poyntz, S. (2011). 'But we didn't live in those times': Canadian students negotiate past and present in a time of war. Education as Change, 15(1), 47-62.

Sen, A. (2019). Militarisation of citizenship education curriculum in Turkey. Journal of Peace Education, 16(1), 78-103.

Skaras, M. (2019). Constructing a national narrative in civil war: history teaching and national unity in South Sudan. Comparative Education, 55(4), 517-535.

Stoddard, J. D. (2009). The Ideological Implications of Using "Educational" Film to Teach Controversial Events. Curriculum Inquiry, 39(3), 407-433.

Suárez, D. F. (2008). Rewriting citizenship? Civic education in Costa Rica and Argentina. Comparative Education, 44(4), 485-503.

Teeger, C. (2015). "Both Sides of the Story": History Education in Post-Apartheid South Africa. American Sociological Review, 80(6), 1175-1200.

Thomas, P. (2016). Youth, terrorism and education: Britain's Prevent programme. International Journal of Lifelong Education, 35(2), 171-187.

Toledo Jofré, M., Magendzo Kolstrein, A., Gutiérrez Gianella, V. e Iglesias Segura, R. (2015). Enseñanza de 'temas controversiales' en la asignatura de historia y ciencias sociales desde la perspectiva de los profesores. Estudios pedagógicos (Valdivia), 41(1), 275-292. doi: $10.4067 /$ S0718-07052015000100016

Tse, K. (2001). Society and citizenship education in transition: the case of Macau. International Journal of Educational Development, 21 (4), 305-314.

Usón González, I. (2017). Terrorismo y vulneraciones de Derechos Humanos de motivación política en el caso vasco: estudio exploratorio sobre los conocimientos y la valoración ética de la juventud universitaria vasca. Deusto Journal of Human Rights, 2, 121-148. doi: 10.18543/dihr-2-2017pp121-148

Wills, J. S. (2019). Silencing Racism: Remembering and Forgetting Race and Racism in 11 th-Grade US History Classes. Teachers College Record, $121(4), 040308$.

Zembylas, M., Charalambous, P., Charalambous, C., y Lesta, S. (2016). Human rights and the ethno-nationalist problematic through the eyes of Greek-Cypriot teachers. Education Citizenship and Social Justice, $11(1), 19-33$. 


\section{Anexo I: Artículos analizados que componen la muestra ${ }^{2}$}

1 Aguilar-Forero, N., Mendoza Torres, D. F., Maria Velasquez, A., Felipe Espitia, D., Ducon Pardey, J., \& De Poorter, J. (2019). Global Citizenship Education: A Curricular Innovation in Social Sciences. Revista Internacional De Educacion Para La Justicia Social, 8(2), 89-111. doi:10.15366/riejs2019.8.2.005

2 Akar, B. (2016). Dialogic pedagogies in educational settings for active citizenship, social cohesion and peacebuilding in Lebanon. Education Citizenship and Social Justice, $11(1)$, $44-$ 62. doi: $10.1177 / 1746197915626081$

3 Akar, B., \& Albrecht, M. (2017). Influences of nationalisms on citizenship education: revealing a "dark side' in Lebanon. Nations and Nationalism, 23(3), 547-570. doi: $10.1111 /$ nana. 12316

4 Arias Ferrer, L., Egea Vivancos, A., Sanchez Ibanez, R., Dominguez Castillo, J., Garcia Crespo, F. J., \& Miralles Martinez, P. (2019). Forgotten history or history not taught? The students of Spanish High School and their lack of knowledge about the Civil War. Revista Complutense De Educacion, 30(2), 461-478. doi:10.5209/RCED.57625

5 Arturo ${ }^{2}$ Solano-Solano, J. (2016). The concept of terrorism in schools: Unmasking the official discourse to dispel the myths created in the classroom. Revista Educacion, 40(1) doi: $10.15517 /$ revedu.v40i1.22171

6 Baliqi, B. (2017). The Aftermath of War Experiences on Kosovo's Generation on the Move - Collective Memory and Ethnic Relations among Young Adults in Kosovo. Zeitgeschichte, 44(1), 6-19.

7 Baliqi, B. (2018). Contested war remembrance and ethnopolitical identities in Kosovo. Nationalities Papers-the Journal of Nationalism and Ethnicity, 46(3), 471-483. doi: $10.1080 / 00905992.2017 .1375906$

8 Bartolomei, A. B. (2019). Empowering Youth through Civic and Citizenship Education: The Case of Italy

9 Barton, K. C. (2015). Young adolescents' positioning of human rights: Findings from Colombia, Northern Ireland, Republic of Ireland and the United States. Research in Comparative and International Education, 10(1), 48-70. doi: $10.1177 / 1745499914567819$

10 Baytiyeh, H. (2017). Has the Educational System in Lebanon Contributed to the Growing Sectarian Divisions?. Education and Urban Society, 49(5), 546-559. doi: $10.1177 / 0013124516645163$

11 Bellino, M. J. (2015). The Risks We Are Willing to Take: Youth Civic Development in "Postwar" Guatemala. Harvard Educational Review, 85(4), 537-561. doi:10.17763/00178055.85.4.537

\footnotetext{
2 A pesar de haberse identificado algunos errores en títulos y autores de las citas bibliográficas generadas por WOS, se ha optado por mantener el listado de la muestra tal y como aparece en la base de datos, de cara a favorecer su localización.
} 
12 Bellino, M. J. (2016). So That We Do Not Fall Again History Education and Citizenship in "Postwar" Guatemala. Comparative Education Review, 60(1), 58-79.

13 Bentrovato, D., \& Nissanka, M. (2018). Teaching peace in the midst of civil war: tensions between global and local discourses in Sri Lankan civics textbooks. Global Change Peace \& Security, 30(3), 353-372. doi:10.1080/14781158.2018.1505716

14 Bermudez Velez, A., \& Argumero Martinez, D. (2018). The Narrative Framing of Violence in Teaching Resources about the Spanish Conquest of America. Panta Rei-Revista Digital De Ciencia Y Didactica De La Historia, , 93-1 18. doi:10.6018/pantarei/2018/5

15 Biccum, A. (2018). What Can Counterterrorism Learn from Cognitive Justice in Global Citizenship Education?. International Political Sociology, 12(4), 382-400. doi:10.1093/ips/oly019

16 Bickmore, K., Kaderi, A. S., \& Guerra-Sua, A. (2017). Creating capacities for peacebuilding citizenship: history and social studies curricula in Bangladesh, Canada, Colombia, and Mexico. Journal of Peace Education, 14(3), 282-309. doi: $10.1080 / 17400201.2017 .1365698$

17 Bode, I., \& Heo, S. E. (2017). World War II Narratives in Contemporary Germany and Japan: How University Students Understand Their Past. International Studies Perspectives, 18(2), 131-154. doi:10.1093/isp/ekw011

18 Bravo Sanchez, F. A., \& Ruiz Gomez, L. J. (2017). The use of serious games as an interactive tool to strengthen the meaningful learning of peace education. Ciudad Paz-Ando, 10(2), 7 18. doi: $10.14483 / 2422278 X .11640$

19 Brown, A. L., \& Brown, K. D. (2010). Strange Fruit Indeed: Interrogating Contemporary Textbook Representations of Racial Violence Toward African Americans. Teachers College Record, $112(1), 31-67$.

20 Bruehwiler, I. (2015). Citizenship education in Switzerland before, during and after the First World War. History of Education \& Childrens Literature, 10(1), 99-1 20.

21 Chang, L. (2011). In Muller G. (Ed.), Telling histories of an island nation The academics and politics of history textbooks in contemporary Taiwan.

22 Chowers, E. (2017). Violence and the Hebrew language: Jewish nationalism and the university. Journal of Modern Jewish Studies, 16(3), 358-376. doi: $10.1080 / 14725886.2017 .1295700$

23 Chun, J. (2018). Is it possible to overcome nation-centric histories? Rewriting memories of colonization and war in history textbooks. Interventions-International Journal of Postcolonial Studies, 20(4), 567-585. doi:10.1080/1369801X.2018.1487325

24 Craig, R., \& Davis, V. (2015). In Chandler P. (Ed.), "The only way they knew how to solve their disagreements was to fight". A Textual Analysis of the Indigenous Peoples of North America Before, During and After the Civil Rights' Movement.

25 Cuervo Ballesteros, L. E. (2017). Form for democratic participation. Paths of education for peace. Reflections on education for peace, legal conditions and construction of strategies for the formation of citizen participation in school. Uni-Pluriversidad, 17(2), 39-46. 
26 Darrow, M. H. (2008). In the land of Joan of Arc: The civic education of girls and the prospect of war in France, 1871-1914. French Historical Studies, 31(2), 263-291. doi: $10.1215 / 00161071-2007-022$

27 Darvin, J. (2018). Textual Revolution Reading and Writing about Terrorism and CounterTerrorism in High School Humanities Classes. Critical Education, 9(5)

28 Darweish, M., \& Mohammed, M. A. (2018). History education in schools in Iraqi Kurdistan: representing values of peace and violence. Journal of Peace Education, 15(1), 48-75. doi: $10.1080 / 17400201.2017 .1409198$

29 Davids, N., \& Waghid, Y. (2015). On speaking to violence in post-apartheid schools. Tydskrif Vir Geesteswetenskappe, 55(4), 681-693.

30 Davids, N., \& Waghid, Y. (2016). Higher education as a pedagogical site for citizenship education. Education Citizenship and Social Justice, 11(1), 34-43. doi: $10.1177 / 1746197915626079$

31 de Jesus Mesa-Arango, A., Paz-Palacio, N., \& Rocelia Giraldo-Gil, M. (2019). Citizenship competences and Authority in El Carmen del Darien: case study in an education institutions. Uni-Pluriversidad, 19(1), 33-55. doi:10.17533/udea.unipluri.19.1.03

32 del Pozo Serrano, F. J. (2016). Social School Pedagogy in Colombia: the Model of Universidad Del Norte on School Management and Teaching Training for Citizenship and Peace. Revista Iberoamericana De Educacion, 70, 77-90.

33 Delgado Algarra, E. J., \& Estepa Gimenez, J. (2018). Citizenship and dimensions of memory in the learning of history: analysis of a High School Education case. Vinculos De Historia, 7, 366-388. doi:10.18239/vdh_2018.07.20

34 Diazgranados, S., \& Noonan, J. (2015). The relationship of safe and participatory school environments and supportive attitudes toward violence: Evidence from the Colombian Saber test of Citizenship Competencies. Education Citizenship and Social Justice, 10(1), 79-94. doi: $10.1177 / 1746197914568853$

35 Dillon, E. (2018). Critical History Matters: Understanding Development Education in Ireland Today through the Lens of the Past. Policy \& Practice-a Development Education Review, 27, 14-36.

36 Dvir, Y., Yemini, M., Bronshtein, Y., \& Natur, N. (2018). International education as a novel entity in a public education system: the establishment of a new public international school in Israel. Compare-a Journal of Comparative and International Education, 48(6), 935-953. doi: $10.1080 / 03057925.2017 .1369865$

37 Emerson, A. (2018). The making of the (II)legitimate citizen: the case of the Pakistan Studies textbook. Global Change Peace \& Security, 30(3), 295-311. doi: $10.1080 / 14781158.2018 .1501011$

38 Emerson, A. (2018). Educating Pakistan's Daughters: Girls' Citizenship Education and the Reproduction of Cultural Violence in Pakistan. Studies in Social Justice, 12(2), 291-309.

39 Emkic, E. (2018). Peace Education in Bosnia and Herzegovina. Reconciliation and Education in Bosnia and Herzegovina: from Segregation to Sustainable Peace, 13, 37-49. doi:10.1007/978-3-319-73034-9_3 
40 Espindola, J. (2017). Why Historical Injustice Must be Taught in Schools. Studies in Philosophy and Education, 36(1), 95-106. doi:10.1007/s1 1217-016-9536-1

41 Esteve-Faubel, J., Josephine Martin, T., \& Esteve-Faubel, R. (2019). Protest songs about the Iraq War: An effective trigger for critical reflection?. Education Citizenship and Social Justice, 14(2), 179-195. doi:10.1177/1746197918793003

42 Etxeberria-Mauleon, X. (2018). The presentation of victims in civic education in situations of socio-political transition from violence to peace. Quaestiones Disputatae, 11 (23), 189-217.

43 Eugenia Carrera-Diaz, P. (2018). Research for action, action for research with victims of armed conflict in Soacha, Colombia. Prospectiva, 25, 163-186. doi: $10.25100 /$ prts.v0i25.5966

44 Fallace, T. (2018). American Educators' Confrontation With Fascism. Educational Researcher, 47(1), 46-52. doi:10.3102/0013189X17743726

45 Finkel, S. E., Horowitz, J., \& Rojo-Mendoza, R. T. (2012). Civic Education and Democratic Backsliding in the Wake of Kenya's Post-2007 Election Violence. Journal of Politics, 74(1), 52-65. doi: $10.1017 /$ S0022381611001162

46 Firer, R. (2015). Agents of Obedience Education: the Israeli Jewish History Curriculum for Middle and High Schools. Inted2015: 9th International Technology, Education and Development Conference, 7979-7984.

47 Frisancho, S., \& Reategui, F. (2009). Moral education and post-war societies: the Peruvian case. Journal of Moral Education, 38(4), 421 -443. doi:10.1080/03057240903321907

48 Gordon, D. M. (2010). Disrupting the Master Narrative: Global Politics, Historical Memory, and the Implications for Naturalization Education. Anthropology \& Education Quarterly, 41 (1), 1-17. doi: $10.1111 /$ i.1548-1492.2010.01064.x

49 Goren, H., \& Yemini, M. (2016). Global citizenship education in context: teacher perceptions at an international school and a local Israeli school. Compare-a Journal of Comparative and International Education, 46(5), 832-853. doi:10.1080/03057925.2015.1111752

50 Goren, H., Maxwell, C., \& Yemini, M. (2019). Israeli teachers make sense of global citizenship education in a divided society- religion, marginalisation and economic globalisation. Comparative Education, 55(2), 243-263. doi: $10.1080 / 03050068.2018 .1541660$

51 Gross, M. H. (2014). Struggling to deal with the difficult past: Polish students confront the Holocaust. Journal of Curriculum Studies, 46(4), 441-463. doi: $10.1080 / 00220272.2014 .923513$

52 Guerra-Sua, A. M. (2019). Challenges for Peacebuilding and Citizenship Learning in Colombia. Magis-Revista Internacional De Investigacion En Educacion, 11(23), 169-186. doi: 10.11144 /Javeriana.m 1 1-23.cpcl

53 Helyar, F. (2014). Political partisanship, bureaucratic pragmatism and Acadian nationalism: New Brunswick, Canada's 1920 history textbook controversy. History of Education, 43(1), 72-86. doi: $10.1080 / 0046760 X .2013 .844279$ 
54 Holmberg, U. (2017). 'I was born in the reign...': Historical orientation in Ugandan students' national narratives. London Review of Education, 15(2), 212-226. doi: $10.18546 /$ LRE.15.2.06

55 Huijgen, T., Holthuis, P., van Boxtel, C., van De Grift, W., \& Suhre, C. (2019). Students' historical contextualization and the cold war. British Journal of Educational Studies, 67(4), 439-468. doi:10.1080/00071005.2018.1518512

56 Hunt, S. L. (2017). Mission Bogota: pedagogical governance in a weak state. Citizenship Studies, $21(1)$, 68-84. doi:10.1080/13621025.2016.1252720

57 Jerome, L., \& Elwick, A. (2019) Teaching about terrorism, extremism and radicalisation: some implications for controversial issues pedagogy. Oxford Review of Education, doi: $10.1080 / 03054985.2019 .1667318$

58 Joseph, C. O. (2016). Theatre for peace in East Africa: The quest for 'cosmic equilibrium'. Applied Theatre Research, 4(2), 137-146. doi:10.1386/atr.4.2.137_1

59 Ju-back, S. (2008). "How to Cross the Border" of Historical Perceptions in the History Textbooks of Korea, China and Japan: "Liquidation" of the Asia-Pacific War and Historical Reconciliation. Korea Journal, 48(3), 133-165.

60 Kadiwal, L., \& Durrani, N. (2018). Youth Negotiation of Citizenship Identities in Pakistan: Implications for Global Citizenship Education in Conflict-Contexts. British Journal of Educational Studies, 66(4), 537-558. doi:10.1080/00071005.2018.1533099

61 Keynes, M. (2019). History Education for Transitional Justice? Challenges, Limitations and Possibilities for Settler Colonial Australia. International Journal of Transitional Justice, 13(1), 113-133. doi:10.1093/ijij/ijy026

$62 \mathrm{Kim}, \mathrm{K} .(2010)$. Historical awareness of the post-war generation in Korea and national and social responsibility. Korean Journal of Defense Analysis, 22(4), 435-452. doi: $10.1080 / 10163271.2010 .519928$

63 Korbits, K. (2015). The representation of the Cold War in three Estonian history textbooks. Compare-a Journal of Comparative and International Education, 45(5), 772-791. doi: $10.1080 / 03057925.2014 .917948$

64 Korostelina, K. (2010). War of textbooks: History education in Russia and Ukraine.

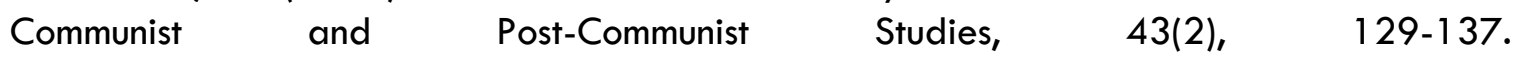
doi: $10.1016 /$ i.postcomstud.2010.03.004

65 Krop, D. S., Holloway, A. G., \& Alberg, D. W. (2012). The USS Monitor: A Pioneering Model for Education and Outreach.

66 Kuppens, L., \& Langer, A. (2018). Can we learn peace at school? An evaluation of the Education for human rights and citizenship (EDHC) course in the post-conflict lvory Coast. International Review of Education, 64(5), 633-650. doi:10.1007/s1 11 159-018-9729-7

67 Lee, J., \& Elwick, A. (2019). Identifying an Educational Response to the Prevent Policy: Student Perspectives on Learning about Terrorism, Extremism and Radicalisation. British Journal of Educational Studies, 67(1), 97-1 14. doi:10.1080/00071005.2017.1415295 
68 Levy, S. A. (2017). How Students Navigate the Construction of Heritage Narratives. Theory and Research in Social Education, 45(2), 157-188. doi: $10.1080 / 00933104.2016 .1240636$

69 Liliana Diaz-Perdomo, M., \& Dario* Rojas-Suarez, N. (2019). Education for Citizenship in the Post-Agreement Times. Eleuthera, 20, 13-34. doi:10.17151/eleu.2019.20.2

70 Lytle, N., \& Floryan, M. (2016). A Design Framework for Experiential History Games. Games and Learning Alliance, Gala 2015, Revised Selected Papers, 9599, 161-170. doi:10.1007/978-3-319-40216-1_17

71 Magendzo, A., \& Isabel Toledo, M. (2009). Moral dilemmas in teaching recent history related to the violation of human rights in Chile. Journal of Moral Education, 38(4), 445465. doi: $10.1080 / 03057240903321923$

72 Mahamud Angulo, K., Groves, T., Milito Barone, C. C., \& Hernandez Laina, Y. (2016). Civic education and visions of war and peace in the Spanish transition to democracy. Paedagogica Historica, 52(1-2), 169-187. doi:10.1080/00309230.2015.1133677

73 Manning, R. (2018). The New Zealand (School Curriculum) 'History Wars': The New Zealand Land Wars Petition and the Status of Maori Histories in New Zealand Schools (1877-2016). Australian Journal of Indigenous Education, 47(2), 120-130. doi:10.1017/iie.2017.13

74 McGhee, D., \& Zhang, S. (2017). Nurturing resilient future citizens through value consistency vs. the retreat from multiculturalism and securitisation in the promotion of British values in schools in the UK. Citizenship Studies, 21(8), 937-950. doi: $10.1080 / 13621025.2017 .1380650$

75 Mmuya, M. (2000). Democratisation, party politics and elections in Tanzania. Tanzania Revisited: Political Stability, Aid Dependency, and Development Constraints, 10, 71-92.

76 Moisan, S. (2019). Teaching history to educate students about human rights? Teaching the history of apartheid in Quebec classrooms. Education Et Francophonie, 47(2), 102-122.

77 Morgan, K. E. (2018). Memory, Truth and the Radicalisation of Knowledge: the Biographies of Nazi Perpetrator Descendants and German History Education. Holocaust-Studii Si Cercetari, 10(11), 243-273.

78 Moss, T. (2019). "Talking Itself Out of a Political Future': Education and Australian Army Engagement with Papua New Guinean Independence, 1966-72. Journal of Pacific History, 54(2), 149-165. doi:10.1080/00223344.2018.1548268

79 Muslih, M. Islamic schooling, migrant Muslims and the problem of integration in The Netherlands. British Journal of Religious Education, doi: $10.1080 / 01416200.2019 .1628004$

80 Mvukiyehe, E., \& Samii, C. (2017). Promoting Democracy in Fragile States: Field Experimental Evidence from Liberia. World Development, 95, 254-267. doi: 10.1016/i.worlddev.2017.02.014

81 Novoa Sanmiguel, D. d. P., \& Escamilla Marquez, D. A. (2019). Start-up of a memoir place in Bucaramanga: The experience of the Oral Archive of the Victims' Memoirs. HallazgosRevista De Investigaciones, 16(31), 41-60. doi:10.15332/s1794-3841.2019.0031.02 
82 Nygren, T., \& Johnsrud, B. (2018). What Would Martin Luther King Jr. Say? Teaching the Historical and Practical Past to Promote Human Rights in Education. Journal of Human Rights Practice, 10(2), 287-306. doi:10.1093/ihuman/huy013

83 Pages, J., \& Marolla, J. (2018). Recent history in the school curricula of Argentina, Chile and Colombia. Challenges of citizenship education from Didactics to the Social Sciences. Historia Y Memoria, 17, 153-184. doi:10.19053/20275137.n17.2018.7455

84 Pardo Mojica, C. (2015). Transpolitical Violence in the Colombian Story: An Analysis of an Emerging Citizenship Through the Story "Album de billetera" by Rodrigo Parra Sandoval. Estudios De Literatura Colombiana, 36, 145-160.

85 Philippou, S. (2009). What makes Cyprus European? Curricular responses of Greek-Cypriot civic education to 'Europe'. Journal of Curriculum Studies, 41(2), 199-223. doi: $10.1080 / 00220270802558406$

86 Philippou, S., \& Theodorou, E. (2019). In Rapoport A. (Ed.), Collapsing the supranational and the national. From Citizenship to Health Education in the Republic of Cyprus.

87 Qian, L., Xu, B., \& Chen, D. (2017). Does History Education Promote Nationalism in China? A "Limited Effect' Explanation. Journal of Contemporary China, 26(104), 199-212. doi: $10.1080 / 10670564.2016 .1223103$

88 Quaynor, L. (2015). 'I do not have the means to speak:' educating youth for citizenship in post-conflict Liberia. Journal of Peace Education, 12(1), 15-36. doi: $10.1080 / 17400201.2014 .931277$

89 Rantala, J. (2011). Children as consumers of historical culture in Finland. Journal of Curriculum Studies, 43(4), 493-506. doi:10.1080/00220272.2011.584563

90 Reich, G. A., \& Corning, A. (2017). Anatomy of a belief: The collective memory of African American Confederate soldiers. Historical Encounters-a Journal of Historical Consciousness Historical Cultures and History Education, 4(2), 11-29.

91 Reilly, J., \& Niens, U. (2014). Global citizenship as education for peacebuilding in a divided society: structural and contextual constraints on the development of critical dialogic discourse in schools. Compare-a Journal of Comparative and International Education, 44(1), 53-76. doi:10.1080/03057925.2013.859894

92 Reyes, M. J., Cruz, M. A., \& Aguirre, F. J. (2016). Places of memory and the new generations: Some political effects of the transmission of memories of Chile's recent past. Revista Espanola De Ciencia Politica-Recp, 41, 93-1 14. doi:10.21308/recp.41.04

93 Reynaud, D., \& Northcote, M. (2015). The World Wars through tabletop wargaming: An innovative approach to university history teaching. Arts and Humanities in Higher Education, 14(4), 349-367. doi:10.1177/1474022214556899

94 Richardson, R. (2015). British values and British identity: Muddles, mixtures, and ways ahead. London Review of Education, 13(2), 37-48. doi:10.18546/LRE.13.2.04

95 Richter, S. (2011). In Muller G. (Ed.), The 'Tokyo Trial view of history' and its revision in contemporary Japan/East Asia. 
96 Rietveld-van Wingerden, M. (2008). Jewish education and identity formation in The Netherlands after the Holocaust. Journal of Beliefs \& Values-Studies in Religion \& Education, 29(2), 185-194. doi:10.1080/13617670802289635

97 Ritiauw, S. P., Maftuh, B., \& Malihah, E. (2017). Model of Conflict Resolution Education Based on Cultural Value of "Pela" in Social Studies Learning. Proceedings of the 1st International Conference on Social Sciences Education: Multicultural Transformation in Education, Social Sciences and Wetland Environment (Icsse 2017), 147, 289-298.

98 Rodriguez Perez, L. C., Bautista, S. M., \& Ramrez Montoya, M. S. (2015). Educational innovation with evidence-based digital portfolios or teaching History. Revista Iberoamericana De Educacion, 69(2), 69-87.

99 Rodriguez-Gomez, D., Foulds, K., \& Sayed, Y. (2016). Representations of Violence in Social Science Textbooks: Rethinking Opportunities for Peacebuilding in the Colombian and South African Post-Conflict Scenarios. Education as Change, 20(3), 76-97. doi:10.17159/1947$9417 / 2016 / 1532$

100 Rubin, B. C. (2016). We Come to Form Ourselves Bit by Bit: Educating for Citizenship in PostConflict Guatemala. American Educational Research Journal, 53(3), 639-672. doi: $10.3102 / 0002831216646871$

101 Russell, S. G., Sirota, S. L., \& Ahmed, A. K. (2019). Human Rights Education in South Africa: Ideological Shifts and Curricular Reforms. Comparative Education Review, 63(1), 1-27. doi: $10.1086 / 701100$

102 Samaie, M., \& Malmir, B. (2017). US news media portrayal of Islam and Muslims: a corpusassisted Critical Discourse Analysis. Educational Philosophy and Theory, 49(14), 1351-1366. doi: $10.1080 / 00131857.2017 .1281789$

103 Seixas, P., Peck, C., \& Poyntz, S. (2011). 'But we didn't live in those times': Canadian students negotiate past and present in a time of war. Education as Change, 15(1), 47-62. doi: $10.1080 / 16823206.2011 .543089$

104 Sen, A. (2019). Militarisation of citizenship education curriculum in Turkey. Journal of Peace Education, 16(1), 78-103. doi:10.1080/17400201.2018.1481019

105 Seyihoglu, A., Sever, R., \& Ozmen, F. (2018). The Current World Problems in the Social Studies and Geography Teacher Candidates' Mind Maps. Marmara Geographical Review, $37,1-15$.

106 Sheppard, M., Kortecamp, K., Jencks, S., Flack, J., \& Wood, A. (2019). Connecting Theory and Practice: Using Place-Based Learning in Teacher Professional Development. Journal of Museum Education, 44(2), 187-200. doi:10.1080/10598650.2019.1597598

107 Sherrod, L., Quinones, O., \& Davila, C. (2004). Youth's political views and their experience of September 11, 2001. Journal of Applied Developmental Psychology, 25(2), 149-170. doi:10.1016/i.appdev.2004.02.002

108 Skaras, M. (2019). Constructing a national narrative in civil war: history teaching and national unity in South Sudan. Comparative Education, 55(4), 517-535. doi: $10.1080 / 03050068.2019 .1634873$ 
109 Skaras, M., \& Breidlid, A. (2016). Teaching the Violent Past in Secondary Schools in Newly Independent South Sudan. Education as Change, 20(3), 98-118. doi:10.17159/1947$9417 / 2016 / 1312$

110 Sotelo Riveros, W. M., \& Gil Quintero, O. A. (2016). Land, Territory and territoriality: antagonisms between the theoretical and the perspective of victims of remove the lands in MapiriOn-Meta y Charras-Guaviare. Ciudad Paz-Ando, 9(1), 153-164.

111 Sousa, R. C. (2015). A Moral and Body Hygiene: Moral and Civic Education, Physical Activities, Sports and Leisure during Military Dictatorship. Cordis-Revista Eletronica De Historia Social Da Cidade, 14, 18-37.

112 Stewart, M. A., \& Walker, K. (2017). English as a Second Language and World War II: Possibilities for Language and Historical Learning. Tesol Journal, 8(1), 44-69. doi: $10.1002 /$ tesj.262

113 Stoddard, J. D. (2009). The Ideological Implications of Using "Educational" Film to Teach Controversial Events. Curriculum Inquiry, 39(3), 407-433. doi:10.1111/i.1467873X.2009.00450.x

114 Suarez, D. F. (2008). Rewriting citizenship? Civic education in Costa Rica and Argentina. Comparative Education, 44(4), 485-503. doi:10.1080/03050060802517505

115 Taylor, E., Taylor, P. C., Karnovsky, S., Aly, A., \& Taylor, N. (2017). "Beyond Bali": a transformative education approach for developing community resilience to violent extremism. Asia Pacific Journal of Education, 37(2), 193-204. doi: $10.1080 / 02188791.2016 .1240661$

116 Teeger, C. (2015). "Both Sides of the Story": History Education in Post-Apartheid South Africa. American Sociological Review, 80(6), 1175-1200. doi: $10.1177 / 0003122415613078$

117 Tezcan, S., Gur, M. S., \& Sanbas, A. (2018). The Turkish War of National Liberation and the Republican Period in Israeli History Textbooks. Bilig, 86, 137-167.

118 Thomas, P. (2016). Youth, terrorism and education: Britain's Prevent programme. International Journal of Lifelong Education, 35(2), 171-187. doi: $10.1080 / 02601370.2016 .1164469$

119 Thorp, R. (2015). Popular history magazines and history education. Historical Encounters-a Journal of Historical Consciousness Historical Cultures and History Education, 2(1), 102-1 12.

120 Tse, K. (2001). Society and citizenship education in transition: the case of Macau. International Journal of Educational Development, 21(4), 305-314. doi:10.1016/S07380593(00)00004-3

121 van Ommering, E. (2015). Formal history education in Lebanon: Crossroads of past conflicts and prospects for peace. International Journal of Educational Development, 41, 200-207. doi:10.1016/i.ijedudev.2014.06.009

122 Waghid, Y. (2009). Education and madrassahs in South Africa: on preventing the possibility of extremism. British Journal of Religious Education, 31(2), 117-128. doi: $10.1080 / 01416200802661142$ 
123 Waldron, F., \& Oberman, R. (2016). Responsible citizens? How children are conceptualised as rights holders in Irish primary schools. International Journal of Human Rights, 20(6), 744 760. doi: $10.1080 / 13642987.2016 .1147434$

124 Wills, J. S. (2019). Silencing Racism: Remembering and Forgetting Race and Racism in 11 thGrade US History Classes. Teachers College Record, $121(4), 040308$.

125 Wineburg, S., Mosborg, S., Porat, D., \& Duncan, A. (2007). Common belief and the cultural curriculum: An intergenerational study of historical consciousness. American Educational Research Journal, 44(1), 40-76. doi:10.3102/0002831206298677

126 Wirkus, L. (2015). An Open Source WebGIS Application for Civic Education on Peace and Conflict. Isprs International Journal of Geo-Information, 4(2), 1013-1032. doi: $10.3390 /$ ijgi4021013

127 Yogev, E. (2012). The image of the 1967 War in Israeli history textbooks as test case: studying an active past in a protracted regional conflict. Oxford Review of Education, 38(2), 171-188. doi: $10.1080 / 03054985.2012 .666031$

128 Zembylas, M., Charalambous, P., Charalambous, C., \& Lesta, S. (2016). Human rights and the ethno-nationalist problematic through the eyes of Greek-Cypriot teachers. Education Citizenship and Social Justice, 11(1), 19-33. doi:10.1177/1746197915626078 